\title{
APOE4 is associated with cognitive and pathological heterogeneity in patients with Alzheimer's disease: a systematic review
}

\author{
Sheina Emrani ${ }^{1}$, Hirra A. Arain ${ }^{2,3}$, Cassandra DeMarshall ${ }^{4}$ and Tal Nuriel ${ }^{2,3^{*}}$ (D)
}

\begin{abstract}
Possession of the $\varepsilon 4$ allele of apolipoprotein $E(A P O E)$ is the primary genetic risk factor for the sporadic form of Alzheimer's disease (AD). While researchers have extensively characterized the impact that APOE $\varepsilon 4$ (APOE4) has on the susceptibility of $A D$, far fewer studies have investigated the phenotypic differences of patients with $A D$ who are APOE4 carriers vs. those who are non-carriers. In order to understand these differences, we performed a qualitative systematic literature review of the reported cognitive and pathological differences between APOE4-positive (APOE4+) vs. APOE4-negative (APOE4-) AD patients. The studies performed on this topic to date suggest that APOE4 is not only an important mediator of AD susceptibility, but that it likely confers specific phenotypic heterogeneity in AD presentation, as well. Specifically, APOE4+ AD patients appear to possess more tau accumulation and brain atrophy in the medial temporal lobe, resulting in greater memory impairment, compared to APOE4- AD patients. On the other hand, APOE4- AD patients appear to possess more tau accumulation and brain atrophy in the frontal and parietal lobes, resulting in greater impairment in executive function, visuospatial abilities, and language, compared to APOE4+ AD patients. Although more work is necessary to validate and interrogate these findings, these initial observations of pathological and cognitive heterogeneity between APOE4+ vs. APOE4- AD patients suggest that there is a fundamental divergence in AD manifestation related to APOE genotype, which may have important implications in regard to the therapeutic treatment of these two patient populations.
\end{abstract}

Keywords: Apolipoprotein E, APOE, APOE4, Alzheimer's disease, AD, Heterogeneity

\section{Introduction}

\section{APOE4 carriers have an increased risk of developing $A D$} In 1993, Roses and colleagues first reported that an individual's risk of developing Alzheimer's disease (AD) is increased if they carry the $\varepsilon 4$ allele of apolipoprotein $\mathrm{E}$ $(A P O E)$ [1-3], an important apolipoprotein that had primarily been studied for its role in transporting cholesterol and other lipids through the periphery and within the brain [4-6]. Since that time, the link between

\footnotetext{
* Correspondence: tn2283@cumc.columbia.edu

${ }^{2}$ Department of Pathology and Cell Biology, Columbia University, 630 West 168th Street, New York, NY 10032, USA

${ }^{3}$ Taub Institute for Research on Alzheimer's Disease and the Aging Brain, Columbia University, 630 West 168th Street, New York, NY 10032, USA Full list of author information is available at the end of the article
}

APOE4 and AD susceptibility has been extensively validated and characterized. A 1997 meta-analysis by Farrer et al. nicely summarizes the general associations between $A P O E$ genotype and $\mathrm{AD}$ susceptibility [7], which has remained relatively consistent in future studies [8-10]. For example, while the APOE2, APOE3, and APOE4 alleles are present in cognitively normal Caucasians at a relative frequency of about $8 \%, 78 \%$, and $14 \%$, respectively, APOE4 has an allele frequency of about $37 \%$ in Caucasian AD patients [7]. When broken down by the specific genotype frequencies, $A P O E 3 / 4$ individuals represent about $21 \%$ of the cognitively normal Caucasian population, vs. about $41 \%$ of Caucasian AD patients (odds ratio [OR] 3.2), whereas APOE4/4 individuals have 
a genotype frequency of about $2 \%$ in the cognitively normal Caucasian population, vs. about $15 \%$ in the $\mathrm{AD}$ affected Caucasian population (OR 14.9) [7]. Furthermore, while possession of the APOE2 allele is protective against $\mathrm{AD}[2,10,11]$, with Caucasian individuals who possess either the $A P O E 2 / 2$ or the APOE2/3 genotype having an OR of 0.6 , this protective effect is overtaken by the risk effect of the APOE4 allele in APOE2/4 individuals (OR 2.6) [7].

These numbers shift, however, when the APOE4-associated risk of $\mathrm{AD}$ is stratified by traits such as age, gender, and ancestry. For example, the effects of the $A P O E 4$ allele on $\mathrm{AD}$ risk are greatest in younger individuals, with the risk of $\mathrm{AD}$ among Caucasian APOE3/4 individuals peaking at age $65(\mathrm{OR} \sim 4)$ and the risk of $\mathrm{AD}$ among Caucasian APOE4/4 individuals peaking at age 60 (OR 15.5) [7]. In terms of gender, numerous studies have found that the effects of APOE4 on AD susceptibility are greater in women than in men $[7,12,13]$, although these gender differences appear to decrease after age 75 [7, 8, 14]. For example, in the Farrer et al. meta-analysis, the authors reported that a 65 -year-old Caucasian woman with an $A P O E 3 / 4$ genotype has an OR of developing AD of over 4, whereas a 65 -year-old Caucasian man with the same genotype has an OR of less than 2 [7]. Perhaps the most intriguing differences in APOE4's effect size, however, are seen in individuals with different ancestral backgrounds. For example, individuals from African-ancestry populations, such as African Americans, have a higher general frequency of APOE4 (APOE4 allele frequency 19\%) than Caucasian populations, but these individuals are at a relatively lower risk of developing $\mathrm{AD}$ (APOE3/4 OR 1.1; APOE4/4 OR 5.7) [7]. However, the opposite appears to be true for East-Asian populations; for example, individuals of Japanese ancestry have a relatively low APOE4 allele frequency $(\sim 9 \%)$, but a relatively high $A P O E 4$-related risk (APOE3/4 OR 5.6; APOE4/4 OR 33.1) [7].

It is clear from these studies that the APOE4 allele is a strong genetic risk factor for developing $\mathrm{AD}$, even though the disease penetrance varies greatly with regard to age, gender, and ancestry. However, while extensive studies have characterized the role of APOE4 in conferring $\mathrm{AD}$ risk, far fewer studies have investigated the effects of APOE4 on the cognitive and pathological manifestation of the disease in individuals who have already converted to $\mathrm{AD}$. In order to understand how a patient's $A P O E$ genotype affects their disease presentation, we have performed a qualitative systematic literature review of the human studies that have been published to date examining the cognitive and pathological differences between APOE4-positive (APOE4+) vs. APOE4-negative (APOE4-) $\mathrm{AD}$ patients. Interestingly, these studies suggest that possession of $A P O E 4$ does in fact result in phenotypic differences between APOE4+ vs. APOE4- AD patients, with APOE4+ AD patients appearing to possess relatively more tau accumulation and brain atrophy in the medial temporal lobe, resulting in greater memory impairment, than APOE4- AD patients, while APOE4- AD patients appear to possess relatively more fronto-parietal lobe tau accumulation and brain atrophy, resulting in greater impairment in executive function, visuospatial abilities, and language, than APOE4+ AD patients (Fig. 1).

\section{Methods}

The data and information utilized in this qualitative systematic review were obtained from literature published between January 1, 1993, and June 1, 2020. A literature search using both electronic and manual search components was performed, with the goal of identifying all studies published during this time period that specifically compared AD presentation in APOE4 carriers vs. APOE4 non-carriers who were diagnosed with $\mathrm{AD}$ using standard methods. In order to accomplish this, PubMed was exhaustively searched to help identify articles containing a combination of keywords: Apoliprotein E, APOE, APOE4, APOE4-positive, APOE4negative, Alzheimer's disease, $A D$, and patients, which was followed by a first level screening of the articles' titles and abstracts to identify studies that directly investigated our review topic. These searches were limited to studies with human subjects that were published in the English language. A manual reference check of the bibliographies of the relevant studies was also performed in order to identify additional articles that were not identified by the electronic search.

The full article of each identified study on this topic was downloaded and stored in a single folder, at which time a second level of screening of the full text was performed to confirm that each article directly compared the cognitive and/or pathological characteristics of $A P O E 4+$ vs. APOE4 - AD patients. Lastly, each study was sorted into one or more of the following diagnostic categories: rate of cognitive decline (17 studies), neuropsychological profile (12 studies), brain atrophy (13 studies), Aß pathology (7 studies), or tau pathology (6 studies). Those studies that did not fit into one of these five categories were not included in the primary review.

We did not exclude studies based on any patient demographic characteristics or any specific methodologies employed. This broad inclusion criterion was utilized in order to provide the scientific community with a comprehensive record of the studies that have investigated the cognitive and pathological differences between APOE4+ vs. APOE4- $\mathrm{AD}$ patients to date. However, the demographic and methodological differences between studies 


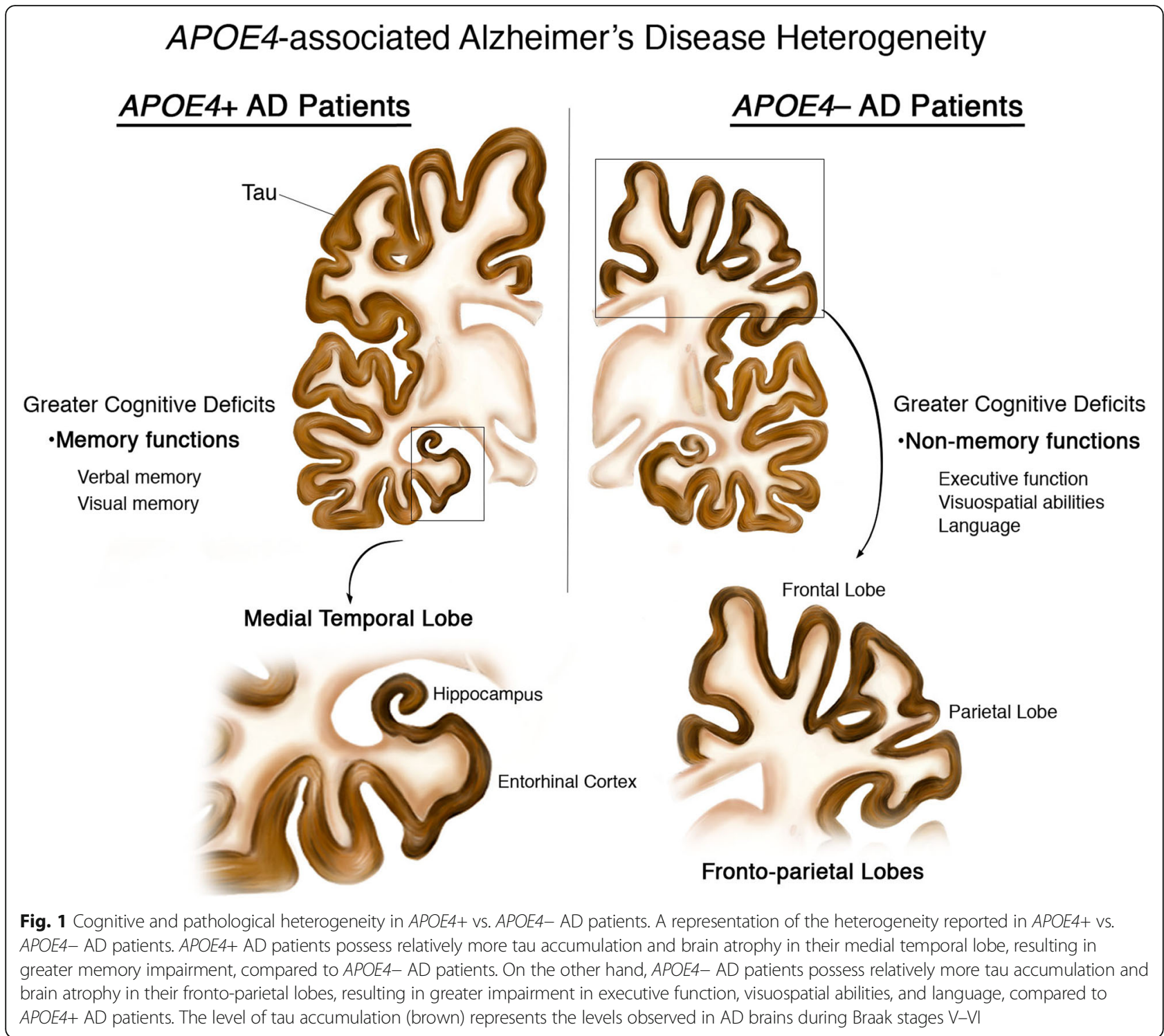

were carefully considered in our overall conclusions, as is discussed throughout the review.

\section{Results}

APOE4+ AD patients do not appear to differ in their overall rates of cognitive decline compared to APOE4- AD patients Although the heterogeneity between APOE4+ vs. APOE4$\mathrm{AD}$ patients is an understudied phenomenon, one question that has been repeatedly investigated over the years is whether or not $A P O E 4+\mathrm{AD}$ patients undergo an accelerated rate of cognitive decline as compared to APOE4- AD patients. However, the results of these studies have been decidedly mixed. While numerous groups have reported that $A P O E 4+\mathrm{AD}$ patients do in fact experience a more accelerated cognitive decline compared to APOE4- AD patients [15-20], other studies have shown either no $A P O E$ genotype-associated differences in the rate of cognitive decline in AD patients [21-27] or slower cognitive decline in $A P O E 4+$ vs. APOE4- AD patients [28-30] (Table 1).

In general, these discrepancies between the reported rates of cognitive decline in APOE4+ vs. APOE4- AD patients highlight the difficulty of trying to determine a consensus about the contributions of one single trait, such as $A P O E$ genotype, on the overall presentation of AD. Adding to this difficulty, each of the studies that we have cited in this review utilizes different methods for their analysis, and the patient populations that they assessed often vary widely in their demographic characteristics. As discussed in the "Introduction" section, differences in age, gender, and ancestral background are known to affect $\mathrm{AD}$ susceptibility among APOE4 carriers; therefore, it is likely that these differences also affect $\mathrm{AD}$ presentation among APOE4 carriers. 
Table 1 Studies investigating the effects of APOE4 on the rate of cognitive decline in AD patients

\begin{tabular}{|c|c|c|}
\hline Study & Study details & Participant details \\
\hline \multicolumn{3}{|c|}{ APOE4 associated with accelerated cognitive decline } \\
\hline $\begin{array}{l}\text { Cosentino } \\
\text { et al. [15] }\end{array}$ & $\begin{array}{l}570 \text { AD patients (WHICAP and Predictors } \\
\text { Study cohorts) were recruited and followed } \\
\text { for an average of } 4 \text { years. Outcome variable } \\
\text { was a composite cognitive } z \text {-score from } \\
\text { five cognitive domains (memory, abstract } \\
\text { reasoning, visuospatial, language, and } \\
\text { executive speed). }\end{array}$ & $\begin{array}{l}\text { Mean age for two population-based } \\
\text { cohorts (WHICAP) and one clinic-based } \\
\text { cohort (Predictors Study) participants was } \\
81.97 \text { ( } n=199 ; 73 \% \text { female, } 61 \% \text { Hispanic, } \\
31 \% \text { African American), } 80.70 \text { ( } n=215 ; 76 \% \\
\text { female, } 62 \% \text { Hispanic, } 28 \% \text { African } \\
\text { American), and } 75.30 \text { ( } n=156 ; 58 \% \text { female, } \\
\text { 0\% Hispanic, 5\% African American) years } \\
\text { of age, respectively. }\end{array}$ \\
\hline
\end{tabular}

Martins et al. $218 \mathrm{AD}$ patients (OPTIMA cohort) were [16] evaluated for cognitive ability using the Cambridge Examination for Mental Disorders of the Elderly (CAMDEX) scale.

Craft et al. [17]

201 probable AD patients were evaluated using the Dementia Rating Scale (DRS) and followed for 1-6years to measure the rate of cognitive decline.

Hirono et al. $64 \mathrm{AD}$ patients were evaluated using [18] the ADAS-Cog and followed for 1 year to measure the rate of cognitive decline.

Kanai et al. $\quad 33$ AD patients were evaluated with the Mini-Mental State Examination (MMSE) and CSF biomarkers, and followed for up to 20 months to measure the rate of cognitive decline.

Chang et al. 104 AD patients (ADNI cohort) and [20] 123 controls were evaluated for changes stratified by age (young-old vs. very-old) and APOE4 status. patients was 70.84 ( $n=49 ; 57 \%$ female), neuropsychological and morphometric
Mean age for APOE4/4 was $71.1(n=28$, $55 \%$ female), APOE3/4 was $73(n=97 ; 54 \%$ female), APOE2/4 was $75.8(n=8 ; 75 \%$ female), APOE3/3 was $75.2(n=69 ; 55 \%$ female), APOE2/3 was $74.2(n=15 ; 53 \%$ female), and APOE2/2 was $79.5(n=1 ; 100 \%$ female). No ancestry information was provided.

Mean age for APOE4/4 was $74.0(n=30$;

$77 \%$ female), APOE3/4 was $78.6(n=82$; $59 \%$ female), APOE3/3 was $79.8(n=75$; $60 \%$ female), and APOE2/3 was $77.3(n=14$; $36 \%$ female). No ancestry information was provided.

Mean age for APOE4+ homozygotes was 70.8 ( $n=8 ; 25 \%$ female), APOE4+

heterozygotes was $73.6(n=33 ; 83 \%$ female), and APOE4- was 76.3 ( $n=23$; $74 \%$ female). No ancestry information was provided.

Mean age for both APOE4+ $(n=17 ; 59 \%$ female) and APOE4- ( $n=16 ; 69 \%$ female) $A D$ patients was 65 . No ancestry information was provided.

Mean age of young-old APOE4+ AD very-old $A P O E 4+A D$ patients was 83.70 ( $n=20 ; 25 \%$ female), young-old APOE4AD patients was 70.53 ( $n=15 ; 53 \%$ female) and very-old APOE4- AD patients was 84.16 ( $n=20 ; 60 \%$ female). No ancestry information was provided.

\section{Study results}

The effect of APOE4 on rate of cognitive decline varied across samples. APOE4+ $A D$ patients in the incident sample demonstrated an accelerated rate of cognitive decline compared to APOE4AD patients. Caucasian participants were more likely to show an association between APOE4 status and rate of cognitive decline as compared to Hispanic and African American participants.

APOE4+ AD patients demonstrated both an earlier and faster rate of cognitive decline compared to APOE4- AD patients. APOE4/4 patients progressed faster than APOE3/4 patients.

APOE4/4 AD patients demonstrated an accelerated rate of cognitive decline compared to APOE4- AD patients.

APOE4+ AD patients demonstrated an accelerated rate of amnestic (assessed by word recall and recognition subtests) and overall cognitive decline, which was significantly correlated with the number of APOE4 alleles.

APOE4+ AD patients demonstrated a more rapid decrease in MMSE score, as well as increased levels of CSF tau compared to APOE4- AD patients.

Young-old ( $\leq 75$ years old) APOE4+ AD patients demonstrated greater cognitive decline in memory and language over a 1 -year interval as compared to other groups, suggesting that the effect of APOE status on rate of decline is dependent upon age at onset of disease.

APOE4 status did not influence the rate of disease progression in either cognitive or functional domains of assessment, regardless of allele dose.

APOE4 status did not influence the rate of cognitive decline across APOE genotypes. $70.3(n=34)$, and APOE4- was $65.5(n=16)$. memory, attention, language, visuosp function, frontal-lobe function, and logica reasoning abilities for up to 5.5 years to measure the rate of cognitive decline.

Holmes et al. 164 late-onset AD patients were evaluated [23] for cognitive and non-cognitive abilities to measure the rate of cognitive decline. Mean age for APOE4+ homozygotes was $68.6(n=16)$, APOE4+ heterozygotes was No ancestry information was provided.

Mean age for APOE4+ homozygotes was 71.6 ( $n=51 ; 70.6 \%$ female), APOE4+ emale), and APOE4- was 73.4 ( $n=156$ $59.6 \%$ female). No ancestry information was provided.

Mean age for APOE4+ AD patients was $75.5(n=92)$ and APOE4- AD patients was $78.7(n=72)$. No ancestry information was provided. heterozygotes was $74.4(n=159 ; 59.1 \%$
APOE4 status was found to be associated with an earlier age of onset, but was not found to influence the cognitive progression of the disease. 
Table 1 Studies investigating the effects of APOE4 on the rate of cognitive decline in AD patients (Continued)

\begin{tabular}{ll}
\hline Study & Study details \\
\hline Kurz et al. & 64 AD patients were evaluated using \\
[24] & the Cambridge Cognitive Examination \\
& (CAMCOG), the MMSE, and the Dementia \\
& Scale (DS) included in the CAMDEX, and \\
& followed for over 3 years to measure the \\
& rate of cognitive decline.
\end{tabular}

Basun et al. 60 late-onset AD patients were evaluated [25] using the MMSE over 3 years to measure the rate of cognitive decline.

Murphy et al. 86 probable AD patients were evaluated [31] using the Mini-Mental State Examination (MMSE) over an average of 3.6 years to measure the rate of cognitive decline.

Farlow et al. $959 \mathrm{AD}$ patients were treated with either [26] metrifonate or placebo for a period of up to 26 weeks to measure the effects of treatment and APOE4 status on cognitive decline using the ADAS-Cog and Clinician's Interview-Based Impression of Change with Caregiver Input (CIBIC-Plus).

Aerssens et al. [27]

1528 probable AD patients were treated with either galantamine or sabeluzole or placebo for a period of up to 1 year to measure the effects of treatment and APOE4 status on cognitive decline using the MMSE and ADAS-cog, along with the Disability Assessment for Dementia (DAD).

\section{APOE4 associated with slower cognitive decline}

Stern et al. 99 probable AD patients (WHICAP cohort) [28] were evaluated using a modified MMSE, as well as other cognitive and motor measures, and were followed biannually for up to 6 years to measure the rate of cognitive decline.

Frisoni et al. 62 sporadic late-onset ( $\geq 70$ years of age) [29] AD patients were evaluated using the MMSE and Clinical Dementia Rating (CDR) to measure the relationship between dis ease progression and APOE4 status.

Hoyt et al. 189 probable AD patients were evaluated [30] using individual growth curve analyses for up to 2 years to measure the rate of cognitive decline using various neuropsychological tests.

\section{Participant details}

The study included 14 males and 50

females, with an average age of 73 .

No ancestry information was provided.

Mean age for APOE4+ AD patients was 81.6 ( $n=27 ; 85 \%$ female) and APOE4AD patients was 85.8 ( $n=33 ; 82 \%$ female). No ancestry information was provided.

Participant details not available.

Mean age (placebo) for APOE4+ homozygotes was 70.5 ( $n=59 ; 62.7 \%$ female, $69.5 \%$ Caucasian), APOE4+ heterozygotes was $74.1(n=183 ; 67.2 \%$ female, $72.1 \%$ Caucasian), and APOE4was $73.2(n=132 ; 57.6 \%$ female, $73.5 \%$ Caucasian). Mean age (metrifonate) for APOE4+ homozygotes was $72.1(n=68$; $57.4 \%$ female, $77.9 \%$ Caucasian), APOE4+ heterozygotes was $74.4(n=281 ; 67.3 \%$ female, 80.4\% Caucasian), and APOE4was 73.5 ( $n=236 ; 61.4 \%$ female, $78.8 \%$ Caucasian).

Mean age was 74.2 (59\% female). No ancestry information was provided.

Mean age for APOE4+ homozygotes was 69.7 ( $n=15 ; 66.7 \%$ female, 80\% Caucasian, 20\% African American), APOE4+ heterozygotes was $71.8(n=41 ; 43.9 \%$ female, 82.9\% Caucasian, 4.9\% African American, and $12.2 \%$ Hispanic), and APOE4 - was 71.3 ( $n=43 ; 44.2 \%$ female, 100\% Caucasian).

Mean age of APOE4+ homozygotes was 78.6 ( $n=19 ; 74 \%$ female), APOE4+ heterozygotes was $79.8(n=16 ; 81 \%$ female), and APOE4- was 80.7 ( $n=27$; $93 \%$ female). No ancestry information was provided.

Mean age for APOE4+ homozygotes was 69.2 ( $n=22 ; 91 \%$ female), APOE4+ heterozygotes was $72.7(n=82 ; 79 \%$ female) and APOE4- was 73.2 ( $n=47$; $68 \%$ female). No ancestry information was provided.

\section{Study results}

APOE4 status was found to have no significant impact on the rate of deterioration in everyday performance, the rate of cognitive decline, or on baseline function and progression of the disease.

APOE4+ AD patients demonstrated a significantly lower age at disease onset and longer duration, but no significant differences were found in the MMSE test scores over time between carriers and non-carriers.

No association between APOE4 allele dosage and rate of cognitive decline was found.

APOE4 genotype in conjunction with metrifonate treatment had no significant effect on global function and cognitive performance in AD patients. APOE4 genotype was not found to influence the rate of disease progression in placebo-treated AD patients.

APOE4/4 AD patients demonstrated a lower age of disease onset, but allele status did not influence the rate of cognitive decline (including in placebo-treated group), or the effectiveness of galantamine treatment.

APOE4+ AD patients demonstrated a lower rate of mortality, slower rate of decline in MMSE scores, less brain atrophy, and a delayed development of myoclonus than APOE4- AD patients. The presence of APOE4 allele was associated with an earlier age of onset of $A D$.

APOE4+ AD patients demonstrated a longer disease duration compared to APOE4- AD patients.

APOE4/4 AD patients demonstrated a slower rate of decline on global cognitive functioning, but not for measures of specific cognitive functions. 
Furthermore, in some of the studies we have cited, the authors have utilized a relatively small number of $\mathrm{AD}$ patients for their analysis; because of this, it is possible that type II statistical errors may affect the conclusions that these authors reported (i.e., a study's small sample size may have resulted in no differences being observed between $A P O E$ genotype groups, even if actual differences exist).

It should also be noted that a large number of the studies investigating the effects $A P O E$ genotype on $\mathrm{AD}$ presentation have focused on "probable" AD patients. Probable $\mathrm{AD}$ is classified using standardized cognitive screening tools and robust neuropsychological tests, and must follow a strict criteria, such as those described by the NINCDSADRDA workgroup in 1984 [32], or an updated criteria described by the NIA-AA workgroup in 2011 [33]. However, the utilization of cognitive profiles alone (or, likewise, the utilization of pathological markers alone) cannot give a $100 \%$ confident diagnosis of AD. Given this information, it is possible that some probable AD patients included in the studies cited in this review were misdiagnosed. Notably, it has been reported that APOE4- individuals make up the majority of $\mathrm{AD}$-diagnosed patients who are later found to be Aß-negative by PET or at autopsy [34, 35]. For this reason, it is possible that the sole reliance on probable AD diagnosis in some of these studies could result in type I statistical errors that may affect their findings (i.e., a study's potential inclusion of non-AD patients, especially if this was weighted towards the APOE4- individuals, may have resulted in significant differences being observed between $A P O E$ genotype groups, even if none exist). Rather than excluding such studies, however, we chose to include them, but to take their limitations into account in our overall, qualitative assessment of the data.

In regard to APOE4's effects on the rate of cognitive decline in $\mathrm{AD}$, assessing the demographic and methodological differences between the studies listed above does provide some clarity. For example, in many of these studies, the authors had access to a relatively small number of $\mathrm{AD}$ patients. One potential approach to assess the findings, therefore, is to focus only on the studies with a relatively large number of participants. Interestingly, when we only include the studies that meet a conservative threshold of $n>100 \mathrm{AD}$ patients, there are three studies that reported accelerated cognitive decline in APOE4+ vs. APOE4- AD patients [15-17], four that reported no difference $[21,23,26,27]$, and only one that reported slower cognitive decline in APOE4+ vs. APOE4- AD patients [30]. Importantly, in the three largest studies from this group, a study by Kleiman et al. that analyzed 366 patients with probable AD [21], a study by Farlow et al. that analyzed 374 placebo-treated $\mathrm{AD}$ clinical-trial participants [26], and a study by Aerssens et al. that analyzed 504 placebo-treated $\mathrm{AD}$ clinical-trial participants [27], the authors did not find any APOE4-associated differences in the rate of cognitive decline in AD. These studies suggest that, when analyzed in a broad fashion, AD patients who carry the APOE4 allele do not appear to possess a more aggressive form of the disease.

However, more work is needed to determine if $A P O E$ genotype may have a significant effect on the rate of cognitive decline in specific subsets of $\mathrm{AD}$ patients, such as within a given age group or gender or ancestry. For example, two of the highly powered studies referenced above, by Cosentino et al. and Craft et al., reported significant APOE4-associated increases in the rates of cognitive decline when looking specifically at incident (i.e., newly diagnosed) $\mathrm{AD}$ cases $[15,17]$. This suggests that $A P O E 4$ may accelerate cognitive decline at the earliest stages of $\mathrm{AD}$ diagnosis, but that these effects may dissipate with increasing disease severity. This possibility would be in line with what occurs prior to $\mathrm{AD}$ diagnosis, where $A P O E 4$ carriers show increased conversion from mild cognitive impairment (MCI) to AD compared to non-carriers [36-38]. Similarly, nondemented elderly $A P O E 4$ carriers have also been reported to undergo increased cognitive decline compared to nondemented elderly non-carriers $[39,40]$, especially when these APOE4 carriers are positive for $A ß$ [41-43].

\section{APOE4+ AD patients have a more amnestic cognitive profile than APOE4- AD patients}

Another factor that deserves critical attention is the multifaceted nature of the cognitive presentation of $\mathrm{AD}$. For example, $\mathrm{AD}$ patients are not only prone to the characteristic amnestic symptoms commonly associated with the disease; they are also prone to deficits in other cognitive domains, such as executive function, visuospatial abilities, and language [44]. Indeed, some atypical $\mathrm{AD}$ patients present with distinct non-amnestic cognitive phenotypes, including corticobasal syndrome (CBS), where patients present with movement impairment; frontal variant Alzheimer's disease (fvAD), where patients present with behavioral/executive function impairment; logopenic variant primary progressive aphasia (lvPPA), where patients present with language impairment; and posterior cortical atrophy (PCA), where patients present with visual impairment. Furthermore, even within the overarching concept of memory, there is significant complexity that must be considered during the neuropsychological assessment of AD patients. For example, poor performance on immediate recall, delayed recall, and delayed recognition is typically suggestive of amnesia [45, 46]. However, difficulties on immediate and delayed recall, in the absence of reduced performance on delayed recognition, are suggestive of problems with lexical access, a task that is associated with significant frontal lobe involvement [47].

In order to assess whether $A P O E$ genotype may alter the cognitive profile of $\mathrm{AD}$ patients, a number of studies have 
utilized neuropsychological assessment tools-including cognitive screening tools, such as the Mini-Mental State Examination (MMSE); brief neuropsychological tests, such as the Alzheimer's Disease Assessment Scale-Cognitive Subscale (ADAS-Cog); or more in-depth neuropsychological tests, such as the California Verbal Learning Test (CVLT) - in an attempt to parse out the potential divergence in cognitive deficits between APOE4+ vs. APOE4$\mathrm{AD}$ patients [48-58]. Interestingly, the majority of these studies have reported that $A P O E 4+\mathrm{AD}$ patients possess relatively more pronounced memory deficits than $A P O E 4$ - AD patients $[49-53,58,59]$, although a few studies did not find an association between $A P O E$ genotype and memory function $[54,55,57]$. In addition, a number of these studies have also reported that APOE4- AD patients possess relatively more pronounced deficits in nonmemory cognitive domains, such as executive function, visuospatial abilities, and language, than $A P O E 4+\mathrm{AD}$ patients $[48,51-58]$, with a greater effect observed in younger APOE4- vs. APOE4+ AD patients [54, 58] (Table 2).

Although these studies utilized different methodological approaches, the results were generally consistent. For example, Scheltens et al. combined four large probable AD cohorts using a neuropsychologically derived cluster analysis and found two distinct groups-a memory-impaired group and a non-memory-impaired group, with the nonmemory-impaired group comprised primarily of younger, APOE4- AD patients, as compared to the memoryimpaired group [48]. Kim et al. recruited 846 South Korean patients diagnosed with probable $\mathrm{AD}$ and categorized them into three groups with respect to their age $(<65$, $65-74$, and $\geq 75$ years old). The authors discovered that younger ( $<65$ years old) APOE4- AD patients performed worse on executive function tasks compared to younger APOE4+ AD patients, while intermediate (65-74 year-old) $A P O E 3 / 4 \mathrm{AD}$ patients performed worse on visuospatial tasks compared to intermediate $A P O E 4 / 4$ AD patients, and older ( $\geq 75$ years old) APOE4/4 AD patients performed worse on verbal memory compared to older APOE4- AD patients [58]. Finally, Wolk et al. compared cognitive differences in 67 APOE4+ vs. 24 APOE4- patients diagnosed with mild $\mathrm{AD}$ and possessing CSF biomarker profiles consistent with AD [51]. APOE4+ AD patients performed worse on memory retention, while APOE4- AD patients were more impaired on tests of working memory, executive function, and lexical access, but not on confrontational naming.

These results suggest that $\mathrm{AD}$ patients likely diverge in their cognitive presentations based on their APOE genotype, with $A P O E 4+\mathrm{AD}$ patients presenting with relatively more pronounced amnestic deficits than APOE4$\mathrm{AD}$ patients, and APOE4- AD patients presenting with relatively more non-memory deficits than $A P O E 4+\mathrm{AD}$ patients. This conclusion is also consistent with the reported observation that $\mathrm{AD}$ patients presenting with atypical phenotypes, such as CBS, fvAD, lvPPA, and PCA, are less likely to be $A P O E 4$ carriers [60, 61].

Interestingly, possession of the APOE4 allele has also been associated with decreased memory performance in non-demented elderly individuals [62-65], as well as with increased incidence of amnestic MCI vs. non-amnestic MCI $[66,67]$. This suggests that possession of the APOE4 allele may confer increased memory deficits throughout the aging to $\mathrm{AD}$ continuum, although it should be noted that APOE4 carriers have also been found to be at an increased risk of developing several non-AD dementias, including vascular dementia (VaD) [68-71], Lewy body dementia (LBD) [72-74], and frontotemporal dementia (FTD) $[75,76]$, which often do not present with a predominantly amnestic phenotype.

\section{APOE4+ AD patients have more atrophy in the medial temporal lobe than APOE4- AD patients}

The cognitive deficits observed in AD patients are a direct result of the pathological abnormalities that occur in a patient's brain during the course of the disease. $\mathrm{AD}$ pathology is characterized by the hallmark accumulation of $A ß$-containing amyloid plaques and hyperphosphorylated tau-containing neurofibrillary tangles (NFTs). Amyloid plaques are extracellular and accumulate in the brain in a rather diffuse manner, typically starting in the neocortex (Thal phase 1), followed by the entorhinal cortex, hippocampus, and insular cortex (Thal phase 2), and eventually accumulating in subcortical regions such as the basal forebrain and brainstem (Thal phases 3-5) [77]. On the other hand, NFTs are intracellular and accumulate in the brain in a more localized and regionally conserved manner, typically occurring first in the transentorhinal and entorhinal cortex regions (Braak stages I-II), followed by the hippocampus and neighboring neocortical regions (Braak stages III-IV), and eventually accumulating throughout the remainder of the neocortex (Braak stages V-IV) [78]. The third major pathological feature of AD is "brain atrophy," as measured by volumetric reduction or cortical thinning observed during magnetic resonance imaging (MRI). In general, the atrophy observed in the brains of $\mathrm{AD}$ patients has been found to follow along the same regional path as NFTs, with the first signs of volumetric loss observed in the medial temporal lobe during the MCI phase, followed by the neocortical portions of the temporal lobe, then the parietal lobe, and finally the frontal lobe during the course of $\mathrm{MCI}$ and $\mathrm{AD}$ progression [79]. In addition to these three distinctive features of $\mathrm{AD}$ pathology, other important pathological events that also occur during the course of the disease include neuroinflammation, deficits in cellular metabolism, cholinergic dysfunction, aberrant network activity, and cerebrovascular pathology [80]. 
Table 2 Studies investigating the effects of APOE4 on cognitive profiles in AD patients

\begin{tabular}{lll}
\hline Study $\quad$ Study details & Participant details & Study results \\
\hline APOE4 associated with more pronounced memory deficits (only) &
\end{tabular}

Marra et al. $\quad 30$ early-onset ( $<65$ years old) and 41 [49] late-onset ( $>70$ years old) AD patients were evaluated for the effects of APOE4 on the age at disease onset.

Snowden et al. $523 \mathrm{AD}$ patients were evaluated to explore [50] the relationship between $A P O E$ status and family history.
Mean age of early-onset APOE4+ AD patients was $58.8(n=20)$, early-onset APOE4- AD patients was $56(n=10)$, late-onset $A P O E 4+A D$ patients was $74.8(n=25)$, and late-onset APOE4AD patients was $76.2(n=16)$. No gender or ancestry information was provided.

Mean age of 60 (56\% female). No ancestry information was provided.

APOE4+ early-onset AD patients exhibited worse performance in measures of learning, long-term verbal memory, and general intelligence tasks. APOE4 status had no effect on cognitive impairment at onset in late-onset $A D$ patients.

APOE4+ AD patients had an older age of onset, a positive family history, and demonstrated greater amnestic deficits than APOE4- AD patients. In contrast, frontal lobe characteristics and posterior cortical presentations were not associated with APOE4 status. In addition, no association was found between reduced age of onset and APOE4 status.

Lehtovirta et al. [53]

Weintraub et al. [59]
58 probable AD patients and 16 controls were evaluated for the effects of age $(<65$ or $\geq 65$ ) and disease type (sporadic or familial) on cognitive decline across various measures.

The APOE genotype of 42 patients with primary progressive aphasia (PPA) and $A D$ pathology (PPA/AD) was compared with 1418 patients with autopsy-confirmed AD and amnestic dementia of the Alzheimer type (DAT/AD).
Mean age of APOE4+ homozygotes was 66 ( $n=13 ; 45 \%$ female), APOE4+ heterozygotes was 72 ( $n=24 ; 46 \%$ female), APOE4- was 70 ( $n=21 ; 52 \%$ female), and control group was 72 ( $n=34 ; 58 \%$ female). No ancestry information was provided.

Mean age of symptom onset for PPA/AD was 60.9 (42.9\% APOE4+, 38.1\% female) and DAT/AD was 68.2 (65.7\% APOE4+, $45.8 \%$ female). No ancestry information was provided.

APOE4+ AD patients demonstrated greater amnestic deficits (immediate and delayed recall) with increasing allele load, and earlier age of onset compared to APOE4$A D$ patients.

DAT/AD patients were found to be enriched for the APOE4 allele, while PPA/AD patients were not.

Across cohorts, AD patients in the non-memory clusters were less often APOE4 carriers and had less severe hippocampal atrophy and more severe posterior cortex atrophy compared to the memory group.

Mean age of APOE4+ AD patients was 65 (46\% female) and APOE4- AD patients was 65 (54\% female). No ancestry information was provided. . the effects of age of onset $(\leq 65$ years old or $>65$ years old) and APOE4 status on cognitive decline.
Davidson et al. $627 \mathrm{mild} / \mathrm{moderate}$ AD patients were [55] evaluated using cognitive screening tools including the MMSE and the Dementia Rating Scale-2 (DRS-2) to identify cognitive subgroups using latent class analysis.

Schott et al. $39 \mathrm{AD}$ patients were assessed using the [56]
MMSE, neuropsychological tests, and MR imaging to investigate $A P O E 4$ frequency in the so-called biparietal AD, characterized as having "combinations of dyscalculia, dyspraxia, visuoperceptual, visuospatial, and spelling deficits with relatively spared memory."
Mean age was 63.4 for males and 63.8 for females (52\% of total subjects were female). All participants were Caucasian.

Mean age of the 10 "biparietal" $A D$ patients was 56.1 ( $60 \%$ female). No ancestry information was provided.
APOE4- AD patients declined faster on language compared to APOE4+ AD patients. When taking age into account, early-onset APOE4- AD patients declined faster on language, attention, executive control, and visuospatial functioning compared to late-onset APOE4+ AD patients. There was no significant difference in decline on memory between groups.

Four classes were generated (Mild, Attention/Construction, Severe, Memory). The Mild class was the most likely to include APOE4+ AD patients, while the Attention/Construction class was least likely to include APOE4+ AD patients.

10 "biparietal AD" patients were identified and were found more likely to be APOE4 non-carriers. 
Table 2 Studies investigating the effects of APOE4 on cognitive profiles in AD patients (Continued)

\begin{tabular}{|c|c|c|c|}
\hline Study & Study details & Participant details & Study results \\
\hline $\begin{array}{l}\text { Hashimoto } \\
\text { et al. [57] }\end{array}$ & $\begin{array}{l}138 \text { probable AD patients were evaluated } \\
\text { for cognitive abilities and regional brain } \\
\text { volume using MRI-based techniques. }\end{array}$ & $\begin{array}{l}\text { Mean age of all three groups, APOE3/3, } \\
\text { APOE3/4, and APOE } 4 / 4 \text {, was } 69(n=46 ; \\
65 \% \text { female for each group). Participants } \\
\text { were of Japanese ancestry. }\end{array}$ & $\begin{array}{l}\text { No significant effects of APOE4 status were } \\
\text { found on memory function, but there was } \\
\text { an association between APOE4- AD } \\
\text { patients and impairment on WMS-R } \\
\text { attention/concentration subtests. Further, } \\
\text { APOE4+ AD patients demonstrated } \\
\text { increased WAIS-R performance and verbal } \\
\text { IQ with increasing allele load compared to } \\
\text { APOE4- AD patients. }\end{array}$ \\
\hline
\end{tabular}

APOE4 associated with more pronounced memory deficits and lack of APOE4 associated with more pronounced non-memory deficits

\author{
Wolk et al. [51] 91 mild AD patients (ADNI cohort) \\ were evaluated for phenotypic differences \\ in cognition and regional cortical volume.
}

Mean age for APOE4+ AD patients was 74 ( $n=67 ; 43 \%$ female) and APOE4- AD patients was 74 ( $n=24 ; 45 \%$ female). No ancestry information was provided.
APOE4+ AD patients demonstrated greater impairment on measures of memory retention, whereas APOE4- AD patients were more impaired on tests of working memory, executive control, and lexical access.

Mean age of APOE4 homozygotes was 66 ( $n=32 ; 65 \%$ female), APOE4 heterozygotes was 66 ( $n=132 ; 56 \%$ female), and APOE4was 67 ( $n=65 ; 51 \%$ female). No ancestry information was provided.

Mean age for the $<65$ group was 58.1 ( $n=184 ; 64 \%$ female), the $65-74$ group was 66.4 ( $n=252 ; 67 \%$ female), and the $\geq$ 75 group was 80.3 ( $n=410 ; 70 \%$ female). Participants were of Korean ancestry.
APOE4+ AD patients demonstrated greater overall amnestic deficits, while APOE4- AD patients were more impaired in domains of naming, executive function, and mental speed.

APOE4- AD patients under 75 years old and APOE3/4 AD patients under 75 years old performed worse on measures of language, visuospatial, and frontal function compared to APOE4/4 AD patients, while APOE $4 / 4$ AD patients over 75 years old performed worse on measures of memory compared to APOE4- AD patients.
With respect to $A P O E$ genotype effects on AD pathology, the most compelling results published to date describe the differing regional patterns of brain atrophy observed in APOE4+ vs. APOE4- AD patients. While a couple of studies have not observed any differences in brain volume or cortical thickness between APOE4+ vs. APOE4- AD patients [81, 82], the vast majority of the studies that have investigated this topic to date have found that $A P O E 4+\mathrm{AD}$ patients possess greater volumetric loss or cortical thinning in the medial temporal lobe than APOE4- $\mathrm{AD}$ patients [51, 57, 58, 83-89], with many reporting that $A P O E 4+$ vs. $A P O E 4-\mathrm{AD}$ patients display volumetric decreases in specific medial temporal lobe structures, such as the hippocampus [57, 83, 86, 87, 89], the amygdala [57, 83, 86, 87], and the entorhinal cortex $[84,89]$. Furthermore, many of these studies also reported that APOE4- $\mathrm{AD}$ patients possess greater volumetric loss or cortical thinning in their frontal and parietal lobes than APOE4+ AD patients [51, 58, 85, 89, 90] (Table 3).

Importantly, a number of these studies noted a direct correlation between the regional brain atrophy patterns that they observed between APOE4+ vs. APOE4- AD patients and the differences in cognitive profile that they observed in these same patients [51, 57, 58, 84, 87, 90]. For example, in the Scheltens et al. study, the authors also analyzed MRI data from their four large probable
$\mathrm{AD}$ cohorts and observed that in their non-memoryimpaired group, which was enriched for APOE4- $\mathrm{AD}$ patients, there was less hippocampal volume loss and more posterior cortex volume loss than in the memoryimpaired group [48]. And in the Kim et al. study, the authors measured cortical thinning using MRI in their 846 South Korean probable AD patients and found that in the younger ( $<65$ years old) APOE4- AD patients, who performed worse on executive function tasks, there was increased bilateral cortical thinning in their lateral frontal, medial frontal, and perisylvian areas compared to the younger APOE4- AD patients, whereas in the older ( $\geq 75$ years old) APOE4+ AD patients, who performed worse on verbal memory tasks, there was increased bilateral cortical thinning in their medial temporal areas compared to the older APOE4- $\mathrm{AD}$ patients [58]. Finally, in the Wolk et al. study, the authors used MRI to measure brain volume and cortical thickness in their mild AD patients and found that APOE4+ $\mathrm{AD}$ patients, who performed worse on memory retention, displayed greater hippocampal volume loss than APOE4- AD patients, whereas APOE4- AD patients, who performed worse on working memory, executive function, and lexical access, displayed decreased cortical thickness in their superior parietal lobule, precuneus, and angular gyrus than $A P O E 4+\mathrm{AD}$ patients. 
Table 3 Studies investigating the effects of APOE4 on brain atrophy in AD patients

\begin{tabular}{|c|c|c|c|}
\hline Study & Study details & Participant details & Study results \\
\hline \multicolumn{4}{|c|}{ No relationship between brain atrophy and APOE4 status } \\
\hline $\begin{array}{l}\text { Drzezga et al. } \\
\text { [81] }\end{array}$ & $\begin{array}{l}32 \text { moderate AD patients matched by } \\
\text { demographics and level of cognitive } \\
\text { impairment were evaluated for brain } \\
\text { volume using cranial MRI and voxel-based } \\
\text { morphometry (VBM). }\end{array}$ & $\begin{array}{l}\text { Mean age for APOE4+ AD patients was } 67 \\
(n=18 ; 50 \% \text { female) and APOE4- AD patients } \\
\text { was } 68 \text { ( } n=14 ; 35 \% \text { female). No ancestry } \\
\text { information was provided. }\end{array}$ & $\begin{array}{l}\text { Comparisons between APOE4+ vs. } \\
\text { APOE4- AD patients showed similar } \\
\text { levels and patterns of brain atrophy }\end{array}$ \\
\hline Jack et al. [82] & $\begin{array}{l}62 \text { probable AD patients and } 125 \text { controls } \\
\text { were evaluated for hippocampal volume } \\
\text { using MRI. }\end{array}$ & $\begin{array}{l}\text { Mean age for both the APOE4+ }(n=36) \text { and } \\
\text { APOE4- }(n=26) \text { AD patients was } 75, \text { while } \\
\text { APOE4+ control group }(n=30) \text { was } 80 \text {, and } \\
\text { APOE4- control group }(n=95) \text { was } 78 \text {. No } \\
\text { ancestry information was provided. }\end{array}$ & $\begin{array}{l}\text { Although the authors noted that } \\
\text { both the AD and control groups } \\
\text { trended towards an APOE4 effect, } \\
\text { there were no significant } \\
\text { differences in hippocampal volume } \\
\text { between APOE4+ vs. APOE4- AD } \\
\text { patients }\end{array}$ \\
\hline
\end{tabular}

\section{APOE4 associated with increased brain atrophy in the medial temporal lobe}

Wolk et al. 91 mild AD cases (ADNI cohort) were

[51] evaluated for cortical volume using MRI morphometric measures.

Hashimoto 138 probable AD patients were evaluated et al. [57] for regional brain volume in the hippocampal formation, amygdaloid complex, and whole brain using MRI-based volumetry techniques.

Kim et al. [58] $846 \mathrm{AD}$ patients and 815 controls were divided into groups based on age $(<65$, $65-74, \geq 75$ years old) to evaluate regional brain volume using MRI

Mattsson et al. [90]

65 Aß-positive AD patients (BioFINDER cohort) were evaluated for tau load and cortical thickness using ${ }^{18} \mathrm{~F}-\mathrm{AV}$-1451 PET and MRI, respectively.

Filippini et al. $83 \mathrm{AD}$ cases were evaluated for regionally [83] specific brain cortical volume using voxel-based morphometry (VBM).

Juottonen

et al. [84]

27 probable AD patients and 31 controls were evaluated for entorhinal cortex volume using MRI.

Pievani et al. 29 AD patients and 29 age- and [85] sex-matched controls were evaluated for cortical volume using MRI.
Mean age for APOE4+ AD patients was 74 ( $n=67 ; 43 \%$ female) and APOE4- AD patients was 74 ( $n=24 ; 45 \%$ female). No ancestry information was provided.

Mean age of all three groups, $A P O E 3 / 3$, APOE3/4, and APOE4/4, was $69(n=46 ; 65 \%$ female for each group). Participants were of Japanese ancestry.

Mean age for the $<65$ group was 58.1 ( $n=184 ; 64 \%$ female), the $65-74$ group was 66.4 ( $n=252 ; 67 \%$ female), and the $\geq 75$ group was 80.3 ( $n=410 ; 70 \%$ female). Participants were of Korean ancestry.

Mean age for APOE4+, AD patients was 72.4 ( $n=46 ; 61 \%$ female) and APOE4- AD patients was 70.1 ( $n=19 ; 53 \%$ female). No ancestry information was provided.

Mean age of APOE4+ homozygotes was 75.5 ( $n=15 ; 80 \%$ female), APOE4+ heterozygotes was 81.1 ( $n=39 ; 53 \%$ female), and APOE4was 75.8 ( $n=29 ; 48 \%$ female). No ancestry information was provided.

Mean age of APOE4+ AD patients was 70 ( $n=16 ; 37 \%$ female), APOE4- AD patients was 69 ( $n=11 ; 54 \%$ female), and control was $72(n=31 ; 64 \%$ female). No ancestry information was provided.

Mean age of APOE4+ AD patients was 71 ( $n=15$; 93\% female), APOE4- AD patients was 68 ( $n=14 ; 50 \%$ female), and control was 69 ( $n=29 ; 72 \%$ female). No ancestry information was provided.

Mean age of APOE4+ homozygotes was 66 ( $n=13 ; 45 \%$ female), APOE4+ heterozygotes was 72 ( $n=24 ; 46 \%$ female), APOE4- was 70 ( $n=21 ; 52 \%$ female), and control group was 72 ( $n=34 ; 58 \%$ female). No ancestry information was provided.
APOE4+ AD patients demonstrated greater brain atrophy in the medial temporal lobe, but less fronto-parietal atrophy compared to APOE4- AD patients.

AD patients demonstrated greater atrophy in the hippocampus and amygdala with increasing APOE4 alleles, whereas whole brain volume increased with increasing APOE4 alleles.

In total $A D$ patients, a higher number of APOE4 alleles were associated with cortical thinning in the bilateral medial temporal areas. Moreover, older $(\geq 75$ years old) APOE4+ AD patients had the most severe medial temporal atrophy, while young ( $<65$ years old) APOE4- AD patients had more severe frontal and perisylvian atrophy.

APOE4- AD patients demonstrated reduced thickness in the lateral and parietal areas compared to APOE4+ $\mathrm{AD}$ patients.

Bilateral medial and anterior temporal lobes, including amygdala, hippocampal, and entorhinal cortex, and orbitofrontal gray matter volume decreased with increasing APOE4 allele load.

APOE4+ AD patients demonstrated greater atrophy in the entorhinal cortex, compared to APOE4- AD patients, with only the left entorhinal cortex reaching statistical significance.

APOE4+ AD patients demonstrated greater brain atrophy in the temporal cortex, right occipital pole, and, to a lesser less degree, in the posterior cingulate, left orbitofrontal and dorsal fronto-parietal cortex compared to APOE4- AD patients.

APOE4+ homozygous AD patients demonstrated greater brain atrophy in the medial temporal structures, hippocampus, and amygdala. However, the frontal lobe volume did not significantly differ between groups. 
Table 3 Studies investigating the effects of APOE4 on brain atrophy in AD patients (Continued)

\begin{tabular}{|c|c|c|c|}
\hline Study & Study details & Participant details & Study results \\
\hline $\begin{array}{l}\text { Lehtovirta } \\
\text { et al. [87] }\end{array}$ & $\begin{array}{l}26 \text { probable AD cases and } 16 \text { age- and } \\
\text { sex-matched controls were evaluated for } \\
\text { hippocampal, amygdala, and frontal lobe } \\
\text { volume using MRI. }\end{array}$ & $\begin{array}{l}\text { Mean age of APOE4/4 AD patients was } 65 \\
\text { ( } n=5 ; 60 \% \text { female), APOE3/4 AD patients } \\
\text { was } 71(n=9 ; 44 \% \text { female), APOE4- (APOE2/ } \\
3 \text { and APOE3/3) AD patients was } 68 \text { ( } n=12 ; \\
41 \% \text { female), while control was } 70 \text { ( } n=16 ; \\
62 \% \text { female). No ancestry information was } \\
\text { provided. }\end{array}$ & $\begin{array}{l}\text { APOE4/4 AD patients had the most } \\
\text { prominent brain atrophy in the } \\
\text { hippocampus and amygdala, and differed } \\
\text { significantly from APOE3/4 and APOE4- } \\
\text { AD patients in the volume of the right } \\
\text { hippocampus and right amygdala. There } \\
\text { were no significant differences between } \\
\text { groups in the frontal lobe. }\end{array}$ \\
\hline $\begin{array}{l}\text { Tanaka et al. } \\
\text { [88] }\end{array}$ & $\begin{array}{l}34 \text { probable AD patients and } 22 \text { controls } \\
\text { were evaluated for morphological and } \\
\text { functional changes using } C T, \text { MRI, and } \\
\text { SPECT. }\end{array}$ & $\begin{array}{l}\text { Mean age of APOE } 4 / 4 \text { AD patients was } 80.8 \\
(n=4) \text {, APOE } 3 / 4 \text { AD patients was } 81(n=8) \text {, } \\
\text { APOE4- (APOE3/3) AD patients was } 84.6 \\
\text { ( } n=22) \text {, while control was } 82 \text { ( } n=22) \text {. No } \\
\text { gender information was provided. } \\
\text { Participants were of Japanese ancestry. }\end{array}$ & $\begin{array}{l}\text { APOE4 allele dose did not affect overall } \\
\text { brain volume during the course of the } \\
\text { disease. However, the inferior temporal } \\
\text { and infero-medial temporal areas were } \\
\text { statistically lower in volume in APOE4+ } \\
\text { AD patients, while the temporal horn } \\
\text { was higher in volume in APOE4+ vs. } \\
\text { APOE4- AD patients. }\end{array}$ \\
\hline $\begin{array}{l}\text { Geroldi et al. } \\
\text { [89] }\end{array}$ & $\begin{array}{l}28 \text { mild to moderate AD patients and } 30 \\
\text { controls were evaluated for hippocampal, } \\
\text { entorhinal cortex, anterior temporal, and } \\
\text { frontal lobe volume using MRI. }\end{array}$ & $\begin{array}{l}\text { Mean age for the AD patients was } 73(n=28 \text {; } \\
78 \% \text { female) while control was } 69(n=30 ; \\
67 \% \text { female). No ancestry information was } \\
\text { provided. }\end{array}$ & $\begin{array}{l}\text { There was increasing atrophy in the } \\
\text { hippocampus, entorhinal cortex, and } \\
\text { anterior temporal lobes with increasing } \\
\text { APOE4 dose. In contrast, larger volumes } \\
\text { of the frontal lobes were observed with } \\
\text { increasing APOE4 dose. }\end{array}$ \\
\hline
\end{tabular}

\section{APOE4+ AD patients do not appear to have higher $A ß$ levels than APOE4- AD patients}

As noted above, the regional brain atrophy that is observed in AD patients is thought to be a direct result of the tau accumulation that progressively occurs in neurons within these brain regions. And it is believed that this tau accumulation and the regional progression of NFTs likely occur downstream of the Aß accumulation/ amyloid plaque deposition that begins early in $\mathrm{AD}$ pathogenesis. Given this information, it is important to determine whether the presentation of these two hallmark pathologies also displays heterogeneity in APOE4+ vs. APOE4- AD patients, and how this presentation may relate to the differences in brain atrophy and cognitive deficits that are observed in these patients. In terms of $\mathrm{A} ß$, it has been well documented that individuals who carry the APOE4 allele accumulate $A ß$ in their brains at an earlier age than non-carriers, and that this occurs long before the onset of AD. For example, a 2015 metaanalysis by Jansen et al. revealed that by the time APOE4/4 carriers turn 40 years old, about $15 \%$ of them will already be positive for cerebral Aß (as detected by PET or CSF), whereas this threshold is not reached until 55 years of age for $A P O E 3 / 4$ carriers and 65 years of age for $A P O E 3 / 3$ carriers [91]. However, $A ß$ levels have been shown to plateau before the clinical diagnosis of $A D$ [92], so any differences in $A ß$ levels associated with $A P O E$ genotype are not expected to be as dramatic once a patient converts to $\mathrm{AD}$ as it is during the linear phase of $A ß$ accumulation. For this reason, it is perhaps not surprising that the handful of studies that have compared the levels of $\mathrm{A} B$ in $A P O E 4+$ vs. APOE4- $\mathrm{AD}$ patients have shown conflicting results, with some studies reporting increased $A ß$ levels in the brains of $A P O E 4+\mathrm{AD}$ patients compared to APOE4- AD patients $[81,93,94]$, some reporting no changes in Aß levels between these two groups $[95,96]$, and some reporting decreased $A ß$ levels in the brains of $A P O E 4+A D$ patients compared to APOE4-AD patients [97, 98] (Table 4).

Looking closely at these studies, it is difficult to make a conclusive statement about how exactly $A P O E$ genotype affects $A ß$ levels or amyloid plaque distribution in the brains of $\mathrm{AD}$ patients. For example, the studies by Drzezga et al. (32 patients with moderate AD) [81], Rowe et al. (53 patients with mild AD) [95], and Lehmann et al. (52 patients with probable AD) [98] each utilized Pittsburgh Compound B (PIB) PET analysis on age- and cognition-matched AD patients who were confirmed to be Aß-positive, but with each study arriving at a different conclusion about the relative levels of $A ß$ in $A P O E 4+$ vs. APOE4 - AD patients. Perhaps future work on this topic will reveal more regionally specific differences in how $A ß$ is distributed in the brains of APOE4+ vs. APOE4$\mathrm{AD}$ patients. This is hinted at by the Lehmann et al. study, where the observed decrease in $\mathrm{A} ß$ in $A P O E 4+$ AD patients was primarily localized to the right lateral frontotemporal regions of the brain [98].

Of course, it is also important to note that amyloid plaques are only one manifestation of $A ß$ pathology that can occur in the brain. $A ß$ can also build up in the walls of arteries (cerebral amyloid angiopathy; $\mathrm{CAA}$ ) or inside of neurons (intraneuronal $\mathrm{A} ß)$. Interestingly, several studies have reported that APOE4+ 
Table 4 Studies investigating the effects of APOE4 on amyloid plaques in AD patients

\begin{tabular}{|c|c|c|}
\hline Study & Study details & Participant details \\
\hline \multicolumn{3}{|c|}{ APOE4 associated with increased amyloid plaques deposition } \\
\hline $\begin{array}{l}\text { Tiraboschi et al. } \\
\text { [93] }\end{array}$ & $\begin{array}{l}296 \text { AD autopsy cases were evaluated } \\
\text { for amyloid plaques and NFTs in the } \\
\text { hippocampus, and midfrontal, inferior } \\
\text { parietal, and superior temporal cortices. }\end{array}$ & $\begin{array}{l}\text { Mean age at death of APOE4+ } \\
\text { homozygotes was } 76.4 \text { ( } n=38 ; 55 \% \\
\text { female), APOE+ heterozygotes was } 80.1 \\
\text { ( } n=149 ; 54 \% \text { female), and APOE4- was } \\
80.2 \text { ( } n=109 ; 58 \% \text { female). No ancestry } \\
\text { information was provided. }\end{array}$ \\
\hline Drzezga et al. [81] & $\begin{array}{l}32 \text { moderate AD patients matched for } \\
\text { demographic and cognitive impairment } \\
\text { were evaluated for amyloid plaque } \\
\text { deposition via PIB-PET imaging. }\end{array}$ & $\begin{array}{l}\text { Mean age for APOE4+ AD patients was } \\
67 \text { ( } n=18 ; 50 \% \text { female) and APOE4-AD } \\
\text { patients was } 68 \text { ( } n=14 ; 35 \% \text { female). } \\
\text { No ancestry information was provided. }\end{array}$ \\
\hline Berg et al. [94] & $\begin{array}{l}186 \text { AD autopsy cases and } 13 \text { controls } \\
\text { were evaluated for multiple brain } \\
\text { histological markers of AD, including brain } \\
\text { densities of amyloid plaques and NFTs. }\end{array}$ & $\begin{array}{l}\text { Broken down by CDR, the mean age at } \\
\text { death of CDR }=0 \text { was } 82.4(n=13 ; 38 \% \\
\text { female), CDR }=0.5 \text { was } 88.6(n=17 ; 52 \% \\
\text { female), CDR }=1 \text { was } 87.8(n=8 ; 50 \% \\
\text { female), CDR }=2 \text { was } 81.2(n=17 ; 52 \% \\
\text { female), and CDR = } 3 \text { was } 79.8(n=144 ; \\
55 \% \text { female). No ancestry information } \\
\text { was provided. }\end{array}$ \\
\hline
\end{tabular}

\section{No relationship between amyloid plaque deposition and APOE4 status}

Rowe et al. [95] 53 mild AD, $57 \mathrm{MCl}$, and 177 control cases (AIBL cohort) were evaluated for amyloid plaque deposition via PIB-PET imaging.

Landen et al. [96] 44 AD, 11 vascular dementia, and 29 age-matched control autopsy cases were evaluated for amyloid plaques and NFTs in the hippocampus and frontal cortex.
Mean age of AD patients was 72.6 ( $n=53 ; 56 \%$ female), $\mathrm{MCl}$ patients was 75.5 ( $n=57 ; 49$ female), and controls was 71.6 ( $n=177 ; 49 \%$ female). No ancestry information was provided.

Mean age at death for $A P O E 4+A D$ patients was $78.1(n=32)$, APOE4-AD patients was $82.5(n=12)$, APOE4+ VaD patients was $76.7(n=3)$, APOE4- VaD patients was $80.1(n=8)$, and APOE4+ controls was $71.0(n=19)$, APOE4controls was $75.7(n=10)$. AD patients were $61 \%$ female, $\mathrm{VaD}$ patients were $27 \%$ female, and controls were $34 \%$ female. No ancestry information was provided.

\section{Study results}

APOE4/4 AD patients demonstrated significantly more amyloid plaques and NFTs in neocortical regions than APOE3/4 or APOE4- AD patients.

APOE4+ AD patients exhibited significantly higher and more extended amyloid plaque deposition, especially in bilateral prefrontal and temporoparietal cortex compared to APOE4- AD patients.

Controlling for dementia severity, plaque densities were weakly associated with APOE4 status in the hippocampus. The degree of CAA was more strongly associated with APOE4 status.

APOE4+ MCl patients and controls exhibited statistically higher PIB binding than APOE4- MCl patients and controls. However, there were no differences observed between APOE4+ vs. APOE4AD patients.

No association was found between APOE4 status and amyloid plaque or NFT levels in either the $A D$, vascular dementia, or control groups.

\section{APOE4 associated with decreased amyloid plaque deposition}

Ossenkoppele et al. [97]

Lehmann et al. [98]
22 APOE4- AD patients, 40 APOE3/4 AD patients, and 22 APOE $4 / 4$ AD patients were evaluated for amyloid plaques and brain metabolism using PIB-PET and FDG-PET, respectively.

52 probable $A D$ and 52 control cases were evaluated for amyloid plaque deposition and brain metabolism using PIB-PET and FDG-PET, respectively.
Mean age of APOE4+ homozygotes was 65 ( $n=22 ; 41 \%$ female), APOE4+ heterozygotes was $62(n=40 ; 38 \%$ female), and APOE4- was 61 ( $n=22 ; 27 \%$ female). No ancestry information was provided.

Mean age for APOE4+ AD patients was 64.3 ( $n=23 ; 48 \%$ female), APOE4- AD patients was 62.7 ( $n=29 ; 41 \%$ female), and controls was 72.3 ( $n=52 ; 58 \%$ female). No ancestry information was provided.
APOE4- AD patients exhibited increased PIB binding in the frontal cortex compared to APOE4+ AD patients, while APOE4- AD patients had less profound metabolic impairment in the posterior parts of the cortex compared to APOE4+ AD patients.

APOE4- AD patients exhibited increased global amyloid plaque burden compared to matched APOE4+ AD patients. In contrast, APOE4+ AD patients exhibited greater medial temporal hypometabolism compared to APOE4- AD patients.
$\mathrm{AD}$ patients have a more frequent CAA comorbidity than APOE4- AD patients [94, 99-101]. As for intraneuronal $A ß$, while one study did report that postmortem brains from APOE4+ AD patients possess higher levels of intraneuronal $A ß$ than those from APOE4- AD patients [102], much more investigation is required before any conclusive statements can be made on this topic.

\section{APOE4+ AD patients appear to develop more tau pathology in their medial temporal lobe than APOE4- AD patients}

As with $A ß$, there have been numerous reports that APOE4 carriers possess higher levels of tau pathology than non-carriers prior to AD onset, although this effect on preclinical tau pathology does not seem to be nearly as robust as it is with $A P O E 4$ 's effects on preclinical $A ß$ 
levels. For example, in a study where Braak and colleagues analyzed autopsied brain tissues specifically from individuals who reached Braak stage I (transentorhinal cortex only) at a relatively young age (less than 47 years old), the authors reported a significant increase in the percentage of $A P O E 4$ carriers in this group (36\%) vs. the percentage of APOE4 carriers in the control group (16\%) [103]. A later, more generalized autopsy study from Braak and colleagues also observed that women who were APOE4 carriers met the criteria for Braak stages II (entorhinal cortex) and III (hippocampus) 3 years earlier than non-carriers [104]. Several more recent studies have also reported a female-dominant effect of $A P O E$ genotype on tau levels prior to $\mathrm{AD}$ diagnosis [12, 105, $106]$. In each of these studies, the authors reported that APOE4 possession increases CSF tau levels specifically in female $A P O E 4$ carriers, with two of the studies reporting that this APOE4-associated effect on CSF tau levels was only present when the women were positive for $A ß$ pathology $[105,106]$.

In regard to $A P O E 4+$ vs. $A P O E 4-\mathrm{AD}$ patients, tau pathology also appears to differ according to $A P O E$

Table 5 Studies investigating the effects of APOE4 on neurofibrillary tangles in AD patients

\begin{tabular}{|c|c|c|c|}
\hline Study & Study details & Participant details & Study results \\
\hline \multicolumn{4}{|c|}{ No relationship between NFT deposition/distribution and APOE4 status } \\
\hline $\begin{array}{l}\text { Petersen et al. } \\
{[107]}\end{array}$ & $\begin{array}{l}94 \mathrm{AD} \text { autopsy cases enriched for atypical } \\
\mathrm{AD} \text { presentation were evaluated for } \\
\text { patterns of regional NFT accumulation in } \\
\text { six selected neocortical and hippocampal } \\
\text { regions. }\end{array}$ & $\begin{array}{l}\text { Age range at death for the entire group } \\
\text { was } 51-73 \text { at age of onset and } 63-86 \text { at } \\
\text { death ( } n=94 ; 40 \% \text { female). No ancestry } \\
\text { information was provided. }\end{array}$ & $\begin{array}{l}\text { No significant difference in regional NFT } \\
\text { density was found between APOE4+ vs. } \\
A P O E 4-A D \text { patients, although there was } \\
\text { a trend ( } p=0.0992) \text { towards more APOE4 } \\
-A D \text { patients in the hippocampal- } \\
\text { sparing group and more APOE+ AD } \\
\text { patients in the limbic predominant group }\end{array}$ \\
\hline
\end{tabular}

\section{APOE4 associated with increased NFT deposition in the medial temporal lobe}

Murray et al. [61] 889 AD autopsy cases were used to study Average age at death for the regional density and distribution of NFTs. Cases were classified as hippocampalsparing, typical, or limbic predominant based on their relative NFT distribution.

Ossenkoppele et al. [108]

Whitwell et al. [109]

Mattsson et al. [90]
20 cases with either $\mathrm{MCl}$ or probable $\mathrm{AD}$ and $15 A B$-negative cognitively normal individuals were evaluated for ${ }^{18} \mathrm{~F}-\mathrm{AV}$ - 145 tau PET ligand uptake, as well as PIB-PET and FDG-PET. uptake in the entorhinal cortex (EC) relative to whole cortex (C). Using $\mathrm{K}$-median cluster analysis, cases were classified into three categories: $\mathrm{EC}^{\mathrm{LO}} / \mathrm{C}^{\mathrm{LO}}$, $\mathrm{EC}^{\mathrm{LO}} / \mathrm{C}^{\mathrm{Hi}}$, and $\mathrm{EC} \mathrm{CH}^{\mathrm{Hi}} / \mathrm{C}^{\mathrm{Hi}}$.

$65 \mathrm{~A}$-positive patients with either $\mathrm{MCl}$ or AD (BioFINDER cohort) were evaluated hippocampal-sparing subtype was 73 ( $n=97 ; 37 \%$ female), typical subtype was 79 ( $n=665 ; 55 \%$ female), and limbic predominant subtype was $86(n=127$; $69 \%$ female). No ancestry information was provided.

Mean age for PCA patients was $63(n=7$; $42 \%$ female), IVPPA patients was $65(n=5$; $80 \%$ female), amnestic AD patients was 67 $(n=5 ; 40 \%$ female), non-amnestic AD patients was 59 ( $n=1 ; 0 \%$ female), behavioral/dysexecutive variant $A D$ patients was 59 ( $n=1 ; 0 \%$ female), and CBS patients was 60 ( $n=1,100 \%$ female). No ancestry information was provided.

Mean age for the $\mathrm{EC}^{\mathrm{LO}} / \mathrm{C}^{\mathrm{Lo}}$ group was 76 ( $n=21 ; 38 \%$ female), the $\mathrm{EC}^{\mathrm{LO}} / \mathrm{C}^{\mathrm{Hi}}$ group was 64 ( $n=21 ; 57 \%$ female), and the $\mathrm{EC}^{\mathrm{Hi}}$ / $C^{\text {Hi }}$ group was 62 ( $n=20 ; 65 \%$ female). No ancestry information was provided. for ${ }^{18} \mathrm{~F}-\mathrm{AV}$-1451 tau PET ligand uptake and cortical thickness via MRI.

Mean age for APOE4+ AD patients was 72.4 ( $n=46 ; 61 \%$ female) and APOE4- AD patients was 70.1 ( $n=19,53 \%$ female). No ancestry information was provided.
Significantly more APOE4+ AD patients were included in the late-onset $(>65$ years old) limbic predominant group, while a trend towards fewer APOE4+ AD patients were included in the hippocampal-sparing group.

APOE4+ AD patients exhibited increased ${ }^{18} \mathrm{~F}-\mathrm{AV}$-1451 uptake in the bilateral medial temporal and right temporoparietal cortex compared to APOE4- AD patients.

APOE4 frequency was found to be significantly lower in the $\mathrm{EC}^{\mathrm{LO}} / \mathrm{C}^{\mathrm{Hi}}$ group (48\%) relative to the $\mathrm{EC}^{\mathrm{LO}} / \mathrm{C}^{\mathrm{Lo}}(84 \%)$ and $\mathrm{EC}^{\mathrm{Hi}} / \mathrm{C}^{\mathrm{Hi}}(74 \%)$ groups. Thus, in the context of high cortical tau load (but not low cortical tau load), fewer APOE4+ AD patients possessed low tau load in the entorhinal cortex compared to APOE4$\mathrm{AD}$ patients.

APOE4+ AD patients exhibited increased tau load in the entorhinal cortex relative to the whole cortex, and lower NFT load in the lateral parietal, medial parietal, occipital, and whole brain cortical areas compared to APOE4- AD patients.

\section{APOE4 associated with increased NFT deposition in other brain regions}

Al-Shaikh et al. 1361 AD subtypes and 103 controls [110]
(FLAME cohort) were assessed for NFT accumulation and neuronal density differences between different $A D$ subtypes (hippocampal-sparing, typical, or limbic predominant)
Mean age at death for the hippocampalsparing subtype was $72(n=175 ; \%$ female), the limbic predominant subtype was 86 ( $n=172 ; 70 \%$ female), the typical subtype was 81 ( $n=1014 ; 54 \%$ female), and controls was 73 ( $n=103 ; 46 \%$ female). No ancestry information was provided.
Within the "typical" AD group, APOE4+ AD patients exhibited higher NFT pathology in their nucleus basalis of Meynert (nbM), located in the basal forebrain, compared to APOE4- AD patients. 
genotype, although the primary differences here appear to revolve around the regional pattern of NFT distribution, as opposed to the overall levels (Table 5). For example, Murray et al. have reported that, when AD autopsy cases were divided into three distinct groups based on the regional pattern of the NFT pathology observed ("hippocampal-sparing," "typical," and "limbic predominant"), there was a trend towards fewer APOE4 carriers in the "hippocampal-sparing" AD group, and there were significantly more late-onset (greater than 65 years old at diagnosis) APOE4 carriers vs. non-carriers in the "limbic predominant" AD group [61]. Although a more recent study failed to replicate this finding in a set of AD autopsy cases enriched for atypical presentation (in which APOE4 carriers were underrepresented), there did appear to be a trend ( $p=0.0992)$ towards more APOE4 carriers among "limbic predominant" AD cases and fewer APOE4 carriers among "hippocampal-sparing" AD cases [107]. Interestingly, a recent follow-up paper by Murray and colleagues also reported that $A P O E 4+$ "typical" $\mathrm{AD}$ patients, as compared to APOE4- "typical" AD patients, possess more NFT pathology in their nucleus basalis of Meynert (nbM), the major source of cholinergic innervation in the brain [110].

To interrogate this correlation between $A P O E$ genotype and tau pathology in living individuals, researchers have begun utilizing recently developed tau PET imaging ligands to compare tau levels in APOE4+ vs. APOE4- AD patients. For example, a small study by Ossenkoppele et al. utilizing the ${ }^{18} \mathrm{~F}-\mathrm{AV}-1451$ tau PET ligand in 20 individuals diagnosed with $\mathrm{MCI}$ or $\mathrm{AD}$ found increased uptake of the PET ligand in bilateral medial temporal and right temporoparietal cortex of APOE4+ patients, as compared to APOE4- patients [108]. And in a study by Whitwell et al. employing ${ }^{18} \mathrm{~F}-\mathrm{AV}$ 1451 to investigate tau deposition in 62 amyloid-positive $\mathrm{AD}$ patients with a mix of typical and atypical $\mathrm{AD}$ presentations, the authors separated their subjects into three groups $\left(\mathrm{EC}^{\mathrm{Lo}} / \mathrm{C}^{\mathrm{Lo}}, \mathrm{EC}^{\mathrm{Lo}} / \mathrm{C}^{\mathrm{Hi}}\right.$, and $\left.\mathrm{EC}^{\mathrm{Hi}} / \mathrm{C}^{\mathrm{Hi}}\right)$ based on the amount of tau deposition they observed in the entorhinal cortex (EC), as compared to the whole cortex (C) [109]. The authors found that the $A P O E 4$ frequency was significantly lower in the $\mathrm{EC}^{\mathrm{Lo}} / \mathrm{C}^{\mathrm{Hi}}$ group, suggesting that APOE4- $\mathrm{AD}$ patients have less relative tau accumulation in the entorhinal cortex region than $A P O E 4+\mathrm{AD}$ patients in the context of high cortical tau load. Finally, in a study by Mattsson et al. that also utilized the ${ }^{18} \mathrm{~F}-\mathrm{AV}-1451$ tau PET ligand, again on a mixed group of $\mathrm{MCI}$ and $\mathrm{AD}$ patients (65 patients total), the authors reported an increased tau load in the entorhinal cortex (relative to the whole cortex) of $A P O E 4+$ patients compared to APOE4- patients, whereas the tau load in the parietal and occipital lobes was higher in APOE4- patients compared to APOE4+ patients [90].

To be clear, these studies on tau pathology in APOE4+ vs. APOE4- AD patients are still somewhat preliminary, with additional work required to confidently answer this question. Specifically, additional tau PET imaging ligand studies are required in pure $\mathrm{AD}$ populations, and with larger sample sizes. Also, as with the other studies on this topic, analysis of specific subgroupings needs to be performed with respect to age, gender, and ancestral background. However, based on these early results, it does appear that APOE4+ $\mathrm{AD}$ patients may possess relatively more NFTs in the medial temporal lobe, most notably in the entorhinal cortex, while APOE4- AD patients may possess more NFTs in other cortical regions, such as the frontal and parietal lobes.

\section{Conclusions}

\section{Primary findings and key limitations}

The majority of the previous research investigating the relationship between APOE4 and AD has focused on elucidating the patterns and mechanisms associated with the increased risk of developing $\mathrm{AD}$ among $A P O E 4$ carriers. And for good reason, after all, APOE4 is the primary genetic risk factor for sporadic AD. However, the possibility that $A P O E 4$ may also affect the cognitive and pathological presentation of $\mathrm{AD}$ deserves significant attention, as this possibility may elucidate differing pathogenic mechanisms between $A P O E 4+$ vs. APOE4- AD patients, both before and after disease onset, and may have important implications for how we should therapeutically treat $A P O E 4+$ vs. APOE4- AD patients.

Overall, the studies that have been performed on this topic to date suggest that APOE4+ vs. APOE4- AD patients do appear to possess both cognitive and pathological heterogeneity in their presentation of the disease, as depicted in Fig. 1. Specifically, the neuropsychological studies outlined above show that APOE4+ AD patients appear to possess relatively more pronounced memory deficits than APOE4- AD patients, while APOE4- AD patients appear to possess relatively more pronounced non-memory deficits (particularly deficits in executive function, visuospatial abilities, and language) than $A P O E 4+\mathrm{AD}$ patients. The literature also points to divergent pathological underpinnings that likely explain the differences in cognitive profiles related to an $A D$ patient's $A P O E$ genotype. Most notably, APOE4+ AD patients appear to possess relatively more brain atrophy in their medial temporal lobe than APOE4- $\mathrm{AD}$ patients, while $A P O E 4-\mathrm{AD}$ patients appear to possess relatively more brain atrophy in their frontal and parietal lobes than $A P O E 4+\mathrm{AD}$ patients. The literature also suggests that the upstream trigger of these regional brain atrophy differences is likely to be the observed differences in the regional distribution of NFTs in APOE4+ vs. APOE4- AD patients, with $A P O E 4+\mathrm{AD}$ patients possessing a greater relative accumulation of NFTs in their medial temporal lobe (particularly in the entorhinal cortex) than APOE4$\mathrm{AD}$ patients, and APOE4- $\mathrm{AD}$ patients possessing 
relatively more NFTs in their frontal and parietal lobes than APOE4+ AD patients. However, due to the limited number of studies performed using recently developed tau PET imaging ligands, this last conclusion is particularly unresolved.

It should also be noted that an AD patient's $A P O E$ genotype may affect the presentation of several additional brain pathologies not covered in the "Results" section of this review. For example, in the previously discussed Lehmann et al. study, where the authors observed regional decreases in $\mathrm{A} ß$ deposition in APOE4+ vs. APOE4- AD patients, the authors also reported regional differences in glucose metabolism (as measured by FDG-PET), with APOE4+ $\mathrm{AD}$ patients displaying more hypometabolism in bilateral medial temporal and right lateral temporal regions than APOE4- AD patients, while APOE4- AD patients displayed more hypometabolism in other cortical areas, including supplementary motor cortex and superior frontal gyrus [98]. Furthermore, autopsied brains from $A P O E 4+$ vs. APOE4-AD patients have also been reported to possess increased levels of two pathological comorbidities commonly associated with AD: TDP-43 [111-113] and Lewy bodies [114, 115].

As noted throughout this review, there are a number of limitations in the studies we cited, which decreases the overall confidence with which we can assert that there is a definitive difference in disease presentation between APOE4+ vs. APOE4- AD patients. For example, some of the studies performed on this topic utilized relatively small sample sizes, which may result in type II ("false-negative") statistical errors. In addition, many of the studies we cited utilized "probable $\mathrm{AD}$ " for their $\mathrm{AD}$ diagnosis, which may result in type I ("false-positive") statistical errors. Lastly, inherent differences among $A P O E 4$ carriers, like age, gender, and ancestral background, are likely to modulate the effects of $A P O E$ genotype on $\mathrm{AD}$ presentation, a possibility that requires much more investigation.

In order to address these issues, we propose that additional studies comparing the cognitive and pathological presentation of $\mathrm{AD}$ in $A P O E 4+$ vs. APOE4- $\mathrm{AD}$ patients should include the following criteria: (1) Comprehensive neuropsychological testing, or numerous cognitive tests measuring multiple cognitive domains, should be utilized to diagnose AD patients. Cognitive screeners, such as the MMSE, are helpful in identifying individuals who require more comprehensive assessment, but robust neuropsychological tests are far more capable of making accurate diagnoses and clear determinations of the severity of a patient's cognitive impairment. (2) Pathological diagnosis should be confirmed using established biomarkers such PET tracers or CSF measurements, or histology on post-mortem tissues if the subjects are deceased. (3) Large, diverse cohorts of $\mathrm{AD}$ patients should be utilized. These cohorts should include hundreds of participants with different ages, genders, and ancestral backgrounds. Power analysis should be performed not only for the cohort as a whole, but also for the individual demographic subgroupings, in order to allow for statistically significant results from each independent subgroup. (4) All three of the first three criteria should be utilized in tandem in order to carefully match the APOE4+ $\mathrm{AD}$ patients to the $A P O E 4-\mathrm{AD}$ patients with which they are being compared. Matched $A P O E$ genotype groups should possess similar neuropsychological profiles, similar pathology levels, and similar demographics, although variations may be necessary depending on the specific question being tested. We anticipate that these robust future studies will definitively determine whether APOE4+ vs. APOE4- AD patients possess the cognitive and pathological heterogeneity that the initial studies on this topic suggest.

\section{Why disease heterogeneity is important}

In recent years, disease heterogeneity has gained increased attention in $\mathrm{AD}$ research, with numerous publications reporting on divergent aspects of $\mathrm{AD}$ such as atypical neuropsychological profiles and mixed pathologies in $\mathrm{AD}$ patients [116-121]. One reason why the topic of disease heterogeneity is so important in $\mathrm{AD}$ research is that it suggests a previously unappreciated complexity that may make therapeutic treatment of $\mathrm{AD}$ more difficult (and could also help to explain past clinical trial failures). If $\mathrm{AD}$ is not the single, uniform disease that researchers once believed it to be, then a single therapeutic strategy may not be able to help all $\mathrm{AD}$ patients equally. In respect to $A P O E$ genotype, disease heterogeneity may even point to divergent pathological mechanisms that will be particularly important to understand when attempting to treat APOE4 carriers vs. non-carriers.

On that topic, there have been numerous examples of therapies showing efficacy in APOE4 carriers, but not in non-carriers, or vice-versa. For example, currently approved acetylcholinesterase inhibitors have often been reported as having differential effects on APOE4+ vs. APOE4- AD patients, although these results have been mixed [122-125]. Investigations of intranasal insulin as an $\mathrm{AD}$ treatment have also shown mixed results, with an acute insulin treatment showing memory improvement in APOE4- MCI and AD patients, but not APOE4+ MCI and AD patients [126], while a chronic insulin treatment has shown memory improvement in $A P O E 4+\mathrm{MCI}$ and $\mathrm{AD}$ patients, but not APOE4- MCI and AD patients [127]. Differential $A P O E$ genotype effects have also been reported for the treatment of mild-to-moderate $\mathrm{AD}$ patients using the diabetes drug rosiglitazone, with APOE4- AD patients, but not $A P O E 4+\mathrm{AD}$ patients, showing cognitive improvement [128]. Furthermore, the retinoid $\mathrm{x}$ receptor (RXR) 
agonist bexarotene has been shown to reduce $A ß$ levels in APOE4- AD patients, but not in APOE4+ AD patients [129]. And in a phase 3 clinical trial of the anti-Aß antibody bapaineuzumab for mild-to-moderate $\mathrm{AD}$, reductions of both $\mathrm{A} ß$ and tau levels were observed in APOE4+ $\mathrm{AD}$ patients, but not in APOE4- AD patients [130].

Given these potential differences in treatment efficacy for $A P O E 4+$ vs. $A P O E 4-\mathrm{AD}$ patients, it is important not only to elucidate any overall cognitive and pathological heterogeneity between these two groups, but also to understand the underlying mechanisms that may drive this heterogeneity. Indeed, the discovery of divergent pathological mechanisms between APOE4+ vs. APOE4AD patients would not only point to important treatment differences for these two patient groups, but it could also help clarify the mechanism of AD pathogenesis in general. The majority of $A D$ research has focused on $A B$ and tau accumulation, the pathological hallmarks of the disease. However, understanding the ways in which differential isoform expression of $A P O E$, which primarily plays a role in cholesterol and lipid trafficking, mediates AD presentation would add important context to how AD develops and progresses.

\section{Potential mechanisms}

For the most part, the studies referenced in this review do not attempt to pinpoint the underlying mechanism(s) responsible for the heterogeneity that they report between $A P O E 4+$ vs. APOE4- AD patients. However, it is worth discussing the potential mechanisms that may be responsible for this heterogeneity. First off, although the pathological differences that occur in APOE4+ vs. APOE4- AD patients appear to center around tau pathology and the resulting brain atrophy, it is still quite possible that the earlier onset of $\mathrm{A} ß$ pathology that occurs in APOE4 carriers vs. non-carriers may play a direct role in the pathological differences that appear to occur in $A P O E 4+$ vs. $A P O E 4-\mathrm{AD}$ patients. If tau aggregation is in fact a direct result of $A ß$ pathology, as is proposed by the amyloid cascade hypothesis, it is probable that the earlier increases in $A ß$ accumulation that are observed in the brains of APOE4 carriers would lead to an early and prolonged accumulation of tau pathology within the entorhinal cortex and hippocampus, the brain regions where NFTs are first observed. This possibility is hinted at by the recent studies showing that women who are positive for both APOE4 and $A ß$ have higher levels of CSF tau compared to other groups $[105,106]$, even in the absence of any cognitive decline [106]. That said, it would be anticipated that this increased early accumulation of tau pathology in the medial temporal lobe of $A P O E 4$ carriers would also translate to increased tau pathology in the fronto-parietal lobes as the disease progresses. However, these initial studies have observed the exact opposite, with decreased tau pathology and neurodegeneration occurring in the fronto-parietal lobes of $A P O E 4+$ vs. APOE4- AD patients.

Alternatively, it is possible that the mechanisms responsible for this observed cognitive and pathological heterogeneity in $A P O E 4+$ vs. APOE4- $\mathrm{AD}$ patients are independent of $A P O E 4$ 's effects on $A ß$. To that end, it is important to note that $A P O E 4$ expression has been found to have a deleterious effect on numerous $\mathrm{A} ß$-independent pathways within the brain, including cholesterol/lipid metabolism [131-133], endosomal-lysosomal processing [134-142], energy metabolism [143-148], neuroinflammation [149-151], and cerebrovascular integrity [152-155]. Furthermore, $A P O E$ appears to be highly expressed in the medial temporal lobe compared to other brain regions, as shown in this spatial modeling of $A P O E$ mRNA levels derived from Allen Brain Atlas mRNA expression data (http://www.meduniwien.ac.at/neuroimaging/lib/dlpage.php?value $=348 \&$ name $=$ apolipoprotein\%20E) [156]. Therefore, cells in the medial temporal lobe of APOE4 carriers may be particularly susceptible to deficits in the biological pathways listed above.

Clearly, there are still many questions left to be answered with regard to this apparent heterogeneity in $A P O E 4+$ vs. APOE4- AD patients, which we anticipate future studies will help to elucidate. We believe that validating and interrogating this $A P O E 4$-associated heterogeneity will yield important information for how best to treat $A D$ patients based on their specific $A P O E$ genotype. In addition, uncovering the biological mechanism(s) responsible for this apparent heterogeneity may pave the way for the discovery of novel therapeutic strategies for treating or preventing $\mathrm{AD}$ in general.

\section{Acknowledgements \\ We acknowledge Drs. Karen Duff and Mark Millan for their editing and advice on this review.}

\section{Authors' contributions}

This review was written by S.E. and T.N., with additional editing and input by H.A.A. and C.D.M. The figure was created by H.A.A., and the tables were created by C.D.M. The authors read and approved the final manuscript.

Authors' information

S.E. is a clinical psychology Ph.D. candidate who trained in the lab of Dr. David Libon at Rowan University. T.N. is an Assistant Professor at Columbia University whose research focuses on APOE4's role in AD.

\section{Funding}

This work was supported by a career development award from the NIA to T.N. (AG061264).

\section{Availability of data and materials}

Not applicable

Ethics approval and consent to participate Not applicable

Consent for publication

Not applicable

Competing interests

The authors declare that they have no competing interests. 


\section{Author details}

'Department of Psychology, Rowan University, 201 Mullica Hill Road, Glassboro, NJ 08028, USA. ²Department of Pathology and Cell Biology, Columbia University, 630 West 168th Street, New York, NY 10032, USA. ${ }^{3}$ Taub Institute for Research on Alzheimer's Disease and the Aging Brain, Columbia University, 630 West 168th Street, New York, NY 10032, USA. ${ }^{4}$ Department of Geriatrics and Gerontology, Rowan University School of Osteopathic Medicine, One Medical Center Drive, Stratford, NJ 08084, USA.

Received: 13 August 2020 Accepted: 22 October 2020 Published online: 04 November 2020

\section{References}

1. Strittmatter WJ, et al. Apolipoprotein E: high-avidity binding to beta-amyloid and increased frequency of type 4 allele in late-onset familial Alzheimer disease. Proc Natl Acad Sci U S A. 1993;90:1977-81.

2. Corder EH, et al. Gene dose of apolipoprotein E type 4 allele and the risk of Alzheimer's disease in late onset families. Science. 1993;261:921-3.

3. Saunders AM, et al. Association of apolipoprotein E allele epsilon 4 with late-onset familial and sporadic Alzheimer's disease. Neurology. 1993;43: 1467-72.

4. Mahley RW, Rall SC Jr. Apolipoprotein E: far more than a lipid transport protein. Annu Rev Genomics Hum Genet. 2000;1:507-37.

5. Han X. The role of apolipoprotein $\mathrm{E}$ in lipid metabolism in the central nervous system. Cell Mol Life Sci. 2004;61:1896-906.

6. Holtzman DM, Herz J, Bu G. Apolipoprotein E and apolipoprotein E receptors: normal biology and roles in Alzheimer disease. Cold Spring Harb Perspect Med. 2012;2:a006312.

7. Farrer $L A$, et al. Effects of age, sex, and ethnicity on the association between apolipoprotein E genotype and Alzheimer disease. A meta-analysis APOE and Alzheimer Disease Meta Analysis Consortium. JAMA. 1997;278:1349-56.

8. Neu SC, et al. Apolipoprotein E genotype and sex risk factors for Alzheimer disease: a meta-analysis. JAMA Neurol. 2017;74:1178-89.

9. Belloy ME, Napolioni V, Greicius MD. A quarter century of APOE and Alzheimer's disease: progress to date and the path forward. Neuron. 2019;101:820-38.

10. Reiman EM, et al. Exceptionally low likelihood of Alzheimer's dementia in APOE2 homozygotes from a 5,000-person neuropathological study. Nat Commun. 2020;11:667.

11. Corder $\mathrm{EH}$, et al. Protective effect of apolipoprotein $\mathrm{E}$ type 2 allele for late onset Alzheimer disease. Nat Genet. 1994;7:180-4.

12. Altmann A, Tian L, Henderson WW, Greicius MD, I. Alzheimer's Disease Neuroimaging Initiative. Sex modifies the APOE-related risk of developing Alzheimer disease. Ann Neurol. 2014;75:563-73.

13. Payami H, et al. Alzheimer's disease, apolipoprotein E4, and gender. JAMA. 1994;271:1316-7.

14. Davidson Y, et al. Apolipoprotein E epsilon4 allele frequency and age at onset of Alzheimer's disease. Dement Geriatr Cogn Disord. 2007;23:60-6

15. Cosentino $\mathrm{S}$, et al. APOE epsilon 4 allele predicts faster cognitive decline in mild Alzheimer disease. Neurology. 2008;70:1842-9.

16. Martins CA, Oulhaj A, de Jager CA, Williams JH. APOE alleles predict the rate of cognitive decline in Alzheimer disease: a nonlinear model. Neurology. 2005;65:1888-93.

17. Craft S, et al. Accelerated decline in apolipoprotein E-epsilon4 homozygotes with Alzheimer's disease. Neurology. 1998;51:149-53.

18. Hirono N, Hashimoto M, Yasuda M, Kazui H, Mori E. Accelerated memory decline in Alzheimer's disease with apolipoprotein epsilon4 allele. J Neuropsychiatry Clin Neurosci. 2003;15:354-8.

19. Kanai $\mathrm{M}$, et al. Apolipoprotein $\mathrm{E} 4$ accelerates dementia and increases cerebrospinal fluid tau levels in Alzheimer's disease. Neurosci Lett. 1999;267:65-8.

20. Chang YL, et al. APOE interacts with age to modify rate of decline in cognitive and brain changes in Alzheimer's disease. Alzheimers Dement. 2014;10:336-48.

21. Kleiman T, et al. Apolipoprotein E epsilon4 allele is unrelated to cognitive or functional decline in Alzheimer's disease: retrospective and prospective analysis. Dement Geriatr Cogn Disord. 2006;22:73-82.

22. Growdon JH, Locascio JJ, Corkin S, Gomez-Isla T, Hyman BT. Apolipoprotein E genotype does not influence rates of cognitive decline in Alzheimer's disease. Neurology. 1996:47:444-8.

23. Holmes C, Levy R, McLoughlin DM, Powell JF, Lovestone S. Apolipoprotein E: non-cognitive symptoms and cognitive decline in late onset Alzheimer's disease. J Neurol Neurosurg Psychiatry. 1996;61:580-3.
24. Kurz A, et al. Apolipoprotein E epsilon 4 allele, cognitive decline, and deterioration of everyday performance in Alzheimer's disease. Neurology. 1996;47:440-3.

25. Basun H, Grut M, Winblad B, Lannfelt L. Apolipoprotein epsilon 4 allele and disease progression in patients with late-onset Alzheimer's disease. Neurosci Lett. 1995;183:32-4.

26. Farlow MR, et al. Metrifonate treatment of AD: influence of APOE genotype. Neurology. 1999:53:2010-6.

27. Aerssens J, et al. APOE genotype: no influence on galantamine treatment efficacy nor on rate of decline in Alzheimer's disease. Dement Geriatr Cogn Disord. 2001;12:69-77.

28. Stern Y, et al. The absence of an apolipoprotein epsilon4 allele is associated with a more aggressive form of Alzheimer's disease. Ann Neurol. 1997;41:615-20.

29. Frisoni $\mathrm{GB}$, et al. Gene dose of the epsilon 4 allele of apolipoprotein $\mathrm{E}$ and disease progression in sporadic late-onset Alzheimer's disease. Ann Neurol. 1995:37:596-604.

30. Hoyt BD, Massman PJ, Schatschneider C, Cooke N, Doody RS. Individual growth curve analysis of APOE epsilon 4-associated cognitive decline in Alzheimer disease. Arch Neurol. 2005;62:454-9.

31. Murphy GM Jr, Taylor J, Kraemer HC, Yesavage J, Tinklenberg JR. No association between apolipoprotein E epsilon 4 allele and rate of decline in Alzheimer's disease. Am J Psychiatry. 1997;154:603-8.

32. McKhann G, et al. Clinical diagnosis of Alzheimer's disease: report of the NINC DS-ADRDA Work Group under the auspices of Department of Health and Human Services Task Force on Alzheimer's Disease. Neurology. 1984;34:939-44.

33. McKhann GM, et al. The diagnosis of dementia due to Alzheimer's disease: recommendations from the National Institute on Aging-Alzheimer's Association workgroups on diagnostic guidelines for Alzheimer's disease. Alzheimers Dement. 2011;7:263-9.

34. Monsell SE, et al. Characterizing apolipoprotein E epsilon4 carriers and noncarriers with the clinical diagnosis of mild to moderate Alzheimer dementia and minimal beta-amyloid peptide plaques. JAMA Neurol. 2015;72:1124-31.

35. Landau SM, Horng A, Fero A, Jagust WJ, I. Alzheimer's Disease Neuroimaging. Amyloid negativity in patients with clinically diagnosed Alzheimer disease and MCl. Neurology. 2016;86:1377-85.

36. Elias-Sonnenschein LS, Viechtbauer W, Ramakers IH, Verhey FR, Visser PJ. Predictive value of APOE-epsilon4 allele for progression from MCl to AD-type dementia: a meta-analysis. J Neurol Neurosurg Psychiatry. 2011;82:1149-56.

37. Fleisher AS, et al. Clinical predictors of progression to Alzheimer disease in amnestic mild cognitive impairment. Neurology. 2007;68:1588-95.

38. Petersen RC, et al. Apolipoprotein E status as a predictor of the development of Alzheimer's disease in memory-impaired individuals. JAMA. 1995:273:1274-8.

39. Caselli RJ, et al. Longitudinal modeling of age-related memory decline and the APOE epsilon4 effect. N Engl J Med. 2009;361:255-63.

40. Caselli RJ, et al. Cognitive domain decline in healthy apolipoprotein $E$ epsilon4 homozygotes before the diagnosis of mild cognitive impairment. Arch Neurol. 2007;64:1306-11.

41. Kantarci $K$, et al. APOE modifies the association between Abeta load and cognition in cognitively normal older adults. Neurology. 2012;78:232-40.

42. Mormino EC, et al. Amyloid and APOE epsilon4 interact to influence shortterm decline in preclinical Alzheimer disease. Neurology. 2014;82:1760-7.

43. Lim YY, et al. Association of beta-amyloid and apolipoprotein E epsilon4 with memory decline in preclinical Alzheimer disease. JAMA Neurol. 2018; 75:488-94.

44. Weintraub S, Wicklund AH, Salmon DP. The neuropsychological profile of Alzheimer disease. Cold Spring Harb Perspect Med. 2012;2:a006171.

45. Davis KL, Price CC, Kaplan E, Libon DJ. Error analysis of the nine-word California Verbal Learning Test (CVLT-9) among older adults with and without dementia. Clin Neuropsychol. 2002;16:81-9.

46. Libon DJ, et al. A nine-word dementia version of the California Verbal Learning Test. Clin Neuropsychol. 1996;10:237-44.

47. McCabe DP, Roediger HL, McDaniel MA, Balota DA, Hambrick DZ. The relationship between working memory capacity and executive functioning: evidence for a common executive attention construct. Neuropsychology. 2010;24:222-43.

48. Scheltens NME, et al. Cognitive subtypes of probable Alzheimer's disease robustly identified in four cohorts. Alzheimers Dement. 2017;13:1226-36.

49. Marra C, et al. Apolipoprotein E epsilon4 allele differently affects the patterns of neuropsychological presentation in early- and late-onset Alzheimer's disease patients. Dement Geriatr Cogn Disord. 2004;18:125-31. 
50. Snowden JS, et al. Cognitive phenotypes in Alzheimer's disease and genetic risk. Cortex. 2007:43:835-45.

51. Wolk DA, Dickerson BC, I. Alzheimer's Disease Neuroimaging. Apolipoprotein E (APOE) genotype has dissociable effects on memory and attentional-executive network function in Alzheimer's disease. Proc Natl Acad Sci U S A. 2010;107:10256-61.

52. van der Vlies $A E$, et al. Cognitive impairment in Alzheimer's disease is modified by APOE genotype. Dement Geriatr Cogn Disord. 2007;24:98-103.

53. Lehtovirta $M$, et al. Clinical and neuropsychological characteristics in familial and sporadic Alzheimer's disease: relation to apolipoprotein E polymorphism. Neurology. 1996;46:413-9.

54. Smits LL, et al. Early onset APOE E4-negative Alzheimer's disease patients show faster cognitive decline on non-memory domains. Eur Neuropsychopharmacol. 2015;25:1010-7

55. Davidson JE, et al. An exploration of cognitive subgroups in Alzheimer's disease. J Int Neuropsychol Soc. 2010;16:233-43.

56. Schott JM, et al. Apolipoprotein e genotype modifies the phenotype of Alzheimer disease. Arch Neurol. 2006;63:155-6.

57. Hashimoto $\mathrm{M}$, et al. Apolipoprotein $\mathrm{E}$ epsilon 4 and the pattern of regiona brain atrophy in Alzheimer's disease. Neurology. 2001;57:1461-6.

58. Kim J, et al. The impact of APOE varepsilon4 in Alzheimer's disease differs according to age. J Alzheimers Dis. 2018;61:1377-85.

59. Weintraub $\mathrm{S}$, et al. APOE is a correlate of phenotypic heterogeneity in Alzheimer disease in a national cohort. Neurology. 2020;94:e607-12.

60. van der Flier WM, Pijnenburg YA, Fox NC, Scheltens P. Early-onset versus late-onset Alzheimer's disease: the case of the missing APOE varepsilon4 allele. Lancet Neurol. 2011;10:280-8.

61. Murray ME, et al. Neuropathologically defined subtypes of Alzheimer's disease with distinct clinical characteristics: a retrospective study. Lancet Neurol. 2011;10:785-96.

62. Lind J, et al. Reduced hippocampal volume in non-demented carriers of the apolipoprotein E epsilon4: relation to chronological age and recognition memory. Neurosci Lett. 2006;396:23-7.

63. Nilsson LG, et al. The influence of APOE status on episodic and semantic memory: data from a population-based study. Neuropsychology. 2006;20: 645-57.

64. Caselli RJ, et al. Longitudinal changes in cognition and behavior in asymptomatic carriers of the APOE e4 allele. Neurology. 2004;62:1990-5.

65. Bondi MW, et al. Episodic memory changes are associated with the APOEepsilon 4 allele in nondemented older adults. Neurology. 1995;45:2203-6.

66. Michaud TL, Su D, Siahpush M, Murman DL. The risk of incident mild cognitive impairment and progression to dementia considering mild cognitive impairment subtypes. Dement Geriatr Cogn Dis Extra. 2017;7:15-29.

67. Knopman DS, et al. Association of prior stroke with cognitive function and cognitive impairment: a population-based study. Arch Neurol. 2009;66:614-9.

68. Rohn TT. Is apolipoprotein E4 an important risk factor for vascular dementia? Int J Clin Exp Pathol. 2014;7:3504-11.

69. Yin YW, et al. Association between apolipoprotein E gene polymorphism and the risk of vascular dementia: a meta-analysis. Neurosci Lett. 2012;514:6-11.

70. Baum L, et al. Apolipoprotein E epsilon4 allele is associated with vascular dementia. Dement Geriatr Cogn Disord. 2006;22:301-5.

71. Frisoni $G B$, et al. Association of apolipoprotein E E4 with vascular dementia. JAMA. 1994;271:1317.

72. Tsuang D, et al. APOE epsilon4 increases risk for dementia in pure synucleinopathies. JAMA Neurol. 2013;70:223-8.

73. Borroni B, et al. APOE genotype and cholesterol levels in Lewy body dementia and Alzheimer disease: investigating genotype-phenotype effect on disease risk. Am J Geriatr Psychiatry. 2006;14:1022-31.

74. Dickson DW, et al. APOE epsilon4 is associated with severity of Lewy body pathology independent of Alzheimer pathology. Neurology. 2018; 91:e1182-95.

75. Koriath $\mathrm{C}$, et al. ApoE4 lowers age at onset in patients with frontotemporal dementia and tauopathy independent of amyloid-beta copathology. Alzheimers Dement (Amst). 2019;11:277-80.

76. Seripa $D$, et al. The APOE gene locus in frontotemporal dementia and primary progressive aphasia. Arch Neurol. 2011;68:622-8.

77. Thal DR, Rub U, Orantes M, Braak H. Phases of A beta-deposition in the human brain and its relevance for the development of AD. Neurology. 2002; 58:1791-800.

78. Braak H, Braak E. Neuropathological stageing of Alzheimer-related changes. Acta Neuropathol. 1991;82:239-59.
79. Whitwell $J$, et al. $3 D$ maps from multiple MRI illustrate changing atrophy patterns as subjects progress from mild cognitive impairment to Alzheimer's disease. Brain. 2007;130:1777-86.

80. Serrano-Pozo A, Frosch MP, Masliah E, Hyman BT. Neuropathological alterations in Alzheimer disease. Cold Spring Harb Perspect Med. 2011;1:a006189.

81. Drzezga A, et al. Effect of APOE genotype on amyloid plaque load and gray matter volume in Alzheimer disease. Neurology. 2009;72:1487-94.

82. Jack CR Jr, et al. Hippocampal atrophy and apolipoprotein E genotype are independently associated with Alzheimer's disease. Ann Neurol. 1998;43: 303-10.

83. Filippini $\mathrm{N}$, et al. Anatomically-distinct genetic associations of APOE epsilon4 allele load with regional cortical atrophy in Alzheimer's disease. Neurolmage. 2009;44:724-8.

84. Juottonen K, Lehtovirta M, Helisalmi S, Riekkinen PJ Sr, Soininen H. Major decrease in the volume of the entorhinal cortex in patients with Alzheimer's disease carrying the apolipoprotein E epsilon4 allele. J Neurol Neurosurg Psychiatry. 1998;65:322-7.

85. Pievani M, et al. Mapping the effect of APOE epsilon4 on gray matter loss in Alzheimer's disease in vivo. Neurolmage. 2009;45:1090-8.

86. Lehtovirta M, et al. SPECT and MRI analysis in Alzheimer's disease: relation to apolipoprotein E epsilon 4 allele. J Neurol Neurosurg Psychiatry. 1996;60:644-9.

87. Lehtovirta $M$, et al. Volumes of hippocampus, amygdala and frontal lobe in Alzheimer patients with different apolipoprotein E genotypes. Neuroscience. 1995;67:65-72.

88. Tanaka S, et al. Inferior temporal lobe atrophy and APOE genotypes in Alzheimer's disease. X-ray computed tomography, magnetic resonance imaging and Xe-133 SPECT studies. Dement Geriatr Cogn Disord. 1998;9:90-8.

89. Geroldi C, et al. APOE-epsilon4 is associated with less frontal and more medial temporal lobe atrophy in AD. Neurology. 1999;53:1825-32.

90. Mattsson N, et al. Greater tau load and reduced cortical thickness in APOE epsilon4-negative Alzheimer's disease: a cohort study. Alzheimers Res Ther. 2018;10:77.

91. Jansen WJ, et al. Prevalence of cerebral amyloid pathology in persons without dementia: a meta-analysis. JAMA. 2015:313:1924-38.

92. Jack CR Jr, et al. Tracking pathophysiological processes in Alzheimer's disease: an updated hypothetical model of dynamic biomarkers. Lancet Neurol. 2013;12:207-16.

93. Tiraboschi $P$, et al. Impact of APOE genotype on neuropathologic and neurochemical markers of Alzheimer disease. Neurology. 2004;62:1977-83.

94. Berg $L$, et al. Clinicopathologic studies in cognitively healthy aging and Alzheimer's disease: relation of histologic markers to dementia severity, age, sex, and apolipoprotein E genotype. Arch Neurol. 1998;55:326-35.

95. Rowe CC, et al. Amyloid imaging results from the Australian Imaging, Biomarkers and Lifestyle (AIBL) study of aging. Neurobiol Aging. 2010;31:1275-83.

96. Landen M, Thorsell A, Wallin A, Blennow K. The apolipoprotein E allele epsilon 4 does not correlate with the number of senile plaques or neurofibrillary tangles in patients with Alzheimer's disease. J Neurol Neurosurg Psychiatry. 1996;61:352-6.

97. Ossenkoppele $\mathrm{R}$, et al. Differential effect of APOE genotype on amyloid load and glucose metabolism in AD dementia. Neurology. 2013;80:359-65.

98. Lehmann $M$, et al. Greater medial temporal hypometabolism and lower cortical amyloid burden in ApoE4-positive AD patients. J Neurol Neurosurg Psychiatry. 2014;85:266-73.

99. Shinohara M, et al. Impact of sex and APOE4 on cerebral amyloid angiopathy in Alzheimer's disease. Acta Neuropathol. 2016;132:225-34.

100. Thal DR, Ghebremedhin E, Orantes M, Wiestler OD. Vascular pathology in Alzheimer disease: correlation of cerebral amyloid angiopathy and arteriosclerosis/lipohyalinosis with cognitive decline. J Neuropathol Exp Neurol. 2003;62:1287-301.

101. Ringman JM, et al. Clinical predictors of severe cerebral amyloid angiopathy and influence of APOE genotype in persons with pathologically verified Alzheimer disease. JAMA Neurol. 2014:71:878-83.

102. Christensen DZ, Schneider-Axmann T, Lucassen PJ, Bayer TA, Wirths $O$. Accumulation of intraneuronal Abeta correlates with ApoE4 genotype. Acta Neuropathol. 2010;119:555-66.

103. Ghebremedhin E, Schultz C, Braak E, Braak H. High frequency of apolipoprotein E epsilon4 allele in young individuals with very mild Alzheimer's diseaserelated neurofibrillary changes. Exp Neurol. 1998;153:152-5.

104. Corder $\mathrm{EH}$, et al. The biphasic relationship between regional brain senile plaque and neurofibrillary tangle distributions: modification by age, sex, and APOE polymorphism. Ann N Y Acad Sci. 2004;1019:24-8. 
105. Hohman TJ, et al. Sex-specific association of apolipoprotein E with cerebrospinal fluid levels of tau. JAMA Neurol. 2018;75:989-98.

106. Buckley RF, et al. Associations between baseline amyloid, sex, and APOE on subsequent tau accumulation in cerebrospinal fluid. Neurobiol Aging. 2019;78:178-85.

107. Petersen $C$, et al. Alzheimer's disease clinical variants show distinct regional patterns of neurofibrillary tangle accumulation. Acta Neuropathol. 2019;138: 597-612.

108. Ossenkoppele $\mathrm{R}$, et al. Tau PET patterns mirror clinical and neuroanatomical variability in Alzheimer's disease. Brain. 2016;139:1551-67.

109. Whitwell JL, et al. [(18) F]AV-1451 clustering of entorhinal and cortical uptake in Alzheimer's disease. Ann Neurol. 2018;83:248-57.

110. Hanna Al-Shaikh FS, et al. Selective vulnerability of the nucleus basalis of Meynert among neuropathologic subtypes of Alzheimer disease. JAMA Neurol. 2019;77:225-33.

111. Wennberg AM, Tosakulwong N, Lesnick TG, Murray ME, Whitwell JL, Liesinger AM, et al. Association of Apolipoprotein E \&4 With Transactive Response DNA-Binding Protein 43. JAMA Neurol. 2018;75(11):1347-1354. https://doi.org/ 10.1001/jamaneurol.2018.3139. PMID: 30422173; PMCID: PMC6248121.

112. Yang HS, et al. Evaluation of TDP-43 proteinopathy and hippocampal sclerosis in relation to APOE epsilon4 haplotype status: a community-based cohort study. Lancet Neurol. 2018;17:773-81.

113. Josephs KA, et al. TDP-43 is a key player in the clinical features associated with Alzheimer's disease. Acta Neuropathol. 2014;127:811-24.

114. Bayram E, Shan G, Cummings JL. Associations between comorbid TDP-43, Lewy body pathology, and neuropsychiatric symptoms in Alzheimer's disease. J Alzheimers Dis. 2019;69:953-61.

115. Chung EJ, et al. Clinical features of Alzheimer disease with and without Lewy bodies. JAMA Neurol. 2015;72:789-96.

116. Emrani S, et al. Alzheimer's/vascular spectrum dementia: classification in addition to diagnosis. J Alzheimers Dis. 2020;73:63-71.

117. Barnes LL, et al. Mixed pathology is more likely in black than white decedents with Alzheimer dementia. Neurology. 2015;85:528-34.

118. James BD, Bennett DA, Boyle PA, Leurgans S, Schneider JA. Dementia from Alzheimer disease and mixed pathologies in the oldest old. JAMA. 2012;307: 1798-800.

119. James BD, et al. TDP-43 stage, mixed pathologies, and clinical Alzheimer'stype dementia. Brain. 2016;139:2983-93.

120. Boyle PA, et al. Person-specific contribution of neuropathologies to cognitive loss in old age. Ann Neurol. 2018;83:74-83.

121. Schneider JA, Arvanitakis Z, Bang W, Bennett DA. Mixed brain pathologies account for most dementia cases in community-dwelling older persons. Neurology. 2007;69:2197-204.

122. Poirier J, et al. Apolipoprotein E4 allele as a predictor of cholinergic deficits and treatment outcome in Alzheimer disease. Proc Natl Acad Sci U S A. 1995;92:12260-4.

123. Waring JF, et al. APOE-varepsilon4 carrier status and donepezil response in patients with Alzheimer's disease. J Alzheimers Dis. 2015;47:137-48.

124. Bizzarro A, et al. Apolipoprotein E epsilon4 allele differentiates the clinical response to donepezil in Alzheimer's disease. Dement Geriatr Cogn Disord. 2005;20:254-61.

125. Petersen RC, et al. Vitamin $\mathrm{E}$ and donepezil for the treatment of mild cognitive impairment. N Engl J Med. 2005;352:2379-88.

126. Reger MA, et al. Effects of intranasal insulin on cognition in memory-impaired older adults: modulation by APOE genotype. Neurobiol Aging. 2006;27:451-8.

127. Claxton $A$, et al. Long acting intranasal insulin detemir improves cognition for adults with mild cognitive impairment or early-stage Alzheimer's disease dementia. J Alzheimers Dis. 2015;45:1269-70.

128. Risner ME, et al. Efficacy of rosiglitazone in a genetically defined population with mild-to-moderate Alzheimer's disease. Pharmacogenomics J. 2006;6:246-54.

129. Cummings $J \mathrm{~L}$, et al. Double-blind, placebo-controlled, proof-of-concept trial of bexarotene Xin moderate Alzheimer's disease. Alzheimers Res Ther. 2016;8:4.

130. Salloway S, et al. Two phase 3 trials of bapineuzumab in mild-to-moderate Alzheimer's disease. N Engl J Med. 2014;370:322-33.

131. Hamanaka $\mathrm{H}$, et al. Altered cholesterol metabolism in human apolipoprotein E4 knock-in mice. Hum Mol Genet. 2000;9:353-61.

132. Chouinard-Watkins R, Plourde M. Fatty acid metabolism in carriers of apolipoprotein E epsilon 4 allele: is it contributing to higher risk of cognitive decline and coronary heart disease? Nutrients. 2014;6:4452-71.

133. Gong JS, et al. Novel action of apolipoprotein E (ApoE): ApoE isoform specifically inhibits lipid-particle-mediated cholesterol release from neurons. Mol Neurodegener. 2007;2:9.
134. DeKroon RM, Armati PJ. The endosomal trafficking of apolipoprotein E3 and E4 in cultured human brain neurons and astrocytes. Neurobiol Dis. 2001;8:78-89.

135. Heeren J, et al. Impaired recycling of apolipoprotein E4 is associated with intracellular cholesterol accumulation. J Biol Chem. 2004;279:55483-92.

136. Ji ZS, et al. Apolipoprotein E4 potentiates amyloid beta peptide-induced lysosomal leakage and apoptosis in neuronal cells. J Biol Chem. 2002;277:21821-8.

137. Ye S, et al. Apolipoprotein (apo) E4 enhances amyloid beta peptide production in cultured neuronal cells: apoE structure as a potential therapeutic target. Proc Natl Acad Sci U S A. 2005;102:18700-5.

138. Li J, et al. Differential regulation of amyloid-beta endocytic trafficking and lysosomal degradation by apolipoprotein E isoforms. J Biol Chem. 2012;287: 44593-601.

139. Yamauchi $K$, et al. Isoform-specific effect of apolipoprotein $E$ on endocytosis of beta-amyloid in cultures of neuroblastoma cells. Ann Clin Lab Sci. 2002; 32:65-74.

140. Rellin L, Heeren J, Beisiegel U. Recycling of apolipoprotein E is not associated with cholesterol efflux in neuronal cells. Biochim Biophys Acta. 2008;1781:232-8.

141. Chen Y, Durakoglugil MS, Xian X, Herz J. ApoE4 reduces glutamate receptor function and synaptic plasticity by selectively impairing ApoE receptor recycling. Proc Natl Acad Sci U S A. 2010;107:12011-6.

142. Nuriel T, et al. The endosomal-lysosomal pathway is dysregulated by APOE4 expression in vivo. Front Neurosci. 2017;11:702.

143. Ong QR, Chan ES, Lim ML, Cole GM, Wong BS. Reduced phosphorylation of brain insulin receptor substrate and Akt proteins in apolipoprotein-E4 targeted replacement mice. Sci Rep. 2014;4:3754.

144. Zhao N, et al. Apolipoprotein E4 impairs neuronal insulin signaling by trapping insulin receptor in the endosomes. Neuron. 2017:96:115-129 e115.

145. Johnson LA, et al. Apolipoprotein E4 mediates insulin resistance-associated cerebrovascular dysfunction and the post-prandial response. J Cereb Blood Flow Metab. 2017;39:770-81.

146. Johnson LA, Torres ER, Impey S, Stevens JF, Raber J. Apolipoprotein E4 and insulin resistance interact to impair cognition and alter the epigenome and metabolome. Sci Rep. 2017;7:43701.

147. Keeney JT, Ibrahimi S, Zhao L. Human ApoE isoforms differentially modulate glucose and amyloid metabolic pathways in female brain: evidence of the mechanism of neuroprotection by ApoE2 and implications for Alzheimer's disease prevention and early intervention. J Alzheimers Dis. 2015;48:411-24.

148. Wu L, Zhang X, Zhao L. Human ApoE isoforms differentially modulate brain glucose and ketone body metabolism: implications for Alzheimer's disease risk reduction and early intervention. J Neurosci. 2018;38:6665-81.

149. Huebbe P, Lodge JK, Rimbach G. Implications of apolipoprotein E genotype on inflammation and vitamin E status. Mol Nutr Food Res. 2010;54:623-30.

150. Rodriguez GA, Tai LM, LaDu MJ, Rebeck GW. Human APOE4 increases microglia reactivity at Abeta plaques in a mouse model of Abeta deposition. J Neuroinflammation. 2014;11:111.

151. Shi Y, et al. ApoE4 markedly exacerbates tau-mediated neurodegeneration in a mouse model of tauopathy. Nature. 2017;549:523-7.

152. Bell RD, et al. Apolipoprotein E controls cerebrovascular integrity via cyclophilin A. Nature. 2012;485:512-6.

153. Tai $L M$, et al. The role of APOE in cerebrovascular dysfunction. Acta Neuropathol. 2016;131:709-23.

154. Mielke MM, et al. Interaction between vascular factors and the APOE epsilon4 allele in predicting rate of progression in Alzheimer's disease. J Alzheimers Dis. 2011;26:127-34.

155. Montagne A, et al. APOE4 leads to blood-brain barrier dysfunction predicting cognitive decline. Nature. 2020;581:71-6.

156. Gryglewski G, et al. Spatial analysis and high resolution mapping of the human whole-brain transcriptome for integrative analysis in neuroimaging. Neurolmage. 2018;176:259-67.

\section{Publisher's Note}

Springer Nature remains neutral with regard to jurisdictional claims in published maps and institutional affiliations. 